\title{
COMMUTING $U$-OPERATORS IN JORDAN ALGEBRAS
}

\author{
JOSÉ A. ANQUELA, TERESA CORTÉS, AND HOLGER P. PETERSSON \\ Abstract. For elements $x, y$ in a non-degenerate non-unital Jordan algebra \\ over a commutative ring, the relation $x \circ y=0$ is shown to imply that the \\ $U$-operators of $x$ and $y$ commute: $U_{x} U_{y}=U_{y} U_{x}$. The proof rests on the \\ $\mathrm{Zel}^{\prime}$ manov-McCrimmon classification of strongly prime quadratic Jordan alge- \\ bras.
}

\section{INTRODUCTION}

In the present paper, we will be concerned with a problem that derives a considerable amount of its significance from the connection between Moufang sets and (quadratic) Jordan division rings established a few years ago by De Medts and Weiss [2]. More specifically, we will focus on a question, raised by K. Tent and communicated to the last-named author by Y. Segev, that may be phrased as follows: given a Jordan division ring with unit $1, U$-operator $U_{x}$ and circle product $x \circ y=U_{x, y} 1$ (see Sections 2.1 and 2.4 below for precise definitions),

(1) does the relation $x \circ y=0$ imply that the linear maps $U_{x}$ and $U_{y}$ commute?

Everyone expecting a short elementary answer to this simple-minded question is in for an unpleasant surprise: the answer we are going to provide in Theorem 4.8 below, though short, and an affirmative one at that, is by no means trivial, relying as it does on a substantial portion of the Zel'manov-McCrimmon classification [15] of arbitrary Jordan division rings. This is all the more regrettable since, from various points of view, it would be desirable to give a proof based exclusively on the manipulation of identities valid in arbitrary Jordan algebras. The hope would then be, for example, that these manipulations could somehow be mimicked in the setting of Moufang sets with abelian root groups, paving the way for new insights into this fascinating topic. On the other hand, they would also show that Question (11) has an affirmative answer not just for division but, in fact, for arbitrary Jordan algebras.

Unfortunately, we have not been able to exhibit a proof of the desired kind. Instead, we must rely on the full arsenal of the Zel'manov-McCrimmon structure theory [15] combined with results of Thedy [20] in order to ensure (11) an affirmative answer for arbitrary non-degenerate, possibly non-unital, Jordan algebras without finiteness conditions (Theorem 9.5). The proof rests on the observation that the answer to (1) is trivially yes for special Jordan algebras (see Section 4.1), combined

Received by the editors August 20, 2012 and, in revised form, December 13, 2012 and December $17,2012$.

2010 Mathematics Subject Classification. Primary 17C10; Secondary 20B22, 20E42, 17C40, $17 \mathrm{C} 60$.

The research of the first two authors was partially supported by the Spanish Ministerio de Economía y Competitividad and Fondos FEDER, MTM2010-16153, and MTM2013-40841-P. 
with the fact that the same conclusion holds for Albert algebras over arbitrary fields (Theorem 8.1); establishing the latter result turns out to be surprisingly delicate. We also obtain affirmative answers for classes of possibly degenerate Jordan algebras, e.g., in the presence of certain algebraicity conditions on the elements involved (Propositions 6.2-6.4), or for Jordan algebras of pointed quadratic forms without 2-torsion over commutative rings (Theorem 7.4 ).

For the convenience of the reader, we have worked hard to keep prerequisites from the theory of Jordan algebras at a minimum; in particular, the standard vocabulary of the theory will be recalled as we go along. Throughout we let $k$ be an arbitrary commutative associative ring of scalars; occasionally it will be replaced by a field which we denote by $F$.

A (non-associative) $k$-algebra $A$ is said to be unital if it contains an identity element $1=1_{A}$, in which case subalgebras of $A$ are said to be unital if they contain the identity $1_{A}$. For a quadratic map $Q: M \rightarrow N$ between $k$-modules, its bilinear polarization will be indicated by $Q(x, y)=Q(x+y)-Q(x)-Q(y)$.

\section{Jordan ALGebras: Generalities}

In this section, we recall some basic facts about arbitrary Jordan algebras that will frequently be used later on. Our main references are Jacobson [6, 7] and McCrimmon-Zel'manov [15].

2.1. The concept of a Jordan algebra. By a (unital) (quadratic) Jordan algebra over $k$ we mean a $k$-module $J$ together with a distinguished element $1:=1_{J} \in J$ (the unit) and a quadratic map $U: J \rightarrow \operatorname{End}_{k}(J), x \mapsto U_{x}$, (the $U$-operator) such that, setting

$$
\{x y z\}:=V_{x, y} z:=U_{x, z} y:=\left(U_{x+z}-U_{x}-U_{z}\right) y
$$

(the Jordan triple product, which is obviously trilinear and symmetric in the outer variables), the following identities hold under all scalar extensions:

$$
\begin{aligned}
U_{1} & =\operatorname{Id}_{J}, \\
U_{x} V_{y, x} & =V_{x, y} U_{x}, \\
U_{U_{x} y} & =U_{x} U_{y} U_{x} .
\end{aligned}
$$

By a Jordan ring we mean a Jordan algebra over $\mathbb{Z}$, the ring of rational integers.

In the remainder of this section, we fix a Jordan algebra $J$ over $k$. A (Jordan) subalgebra of $J$ is a $k$-submodule containing 1 and stable under the operation $U_{x} y$. We define the squaring and its bilinearization in $J$ by

$$
x^{2}=U_{x} 1, \quad x \circ y:=(x+y)^{2}-x^{2}-y^{2} .
$$

Using this, we obtain linear maps $V_{x}: J \rightarrow J$ given by

$$
V_{x} y:=x \circ y
$$

and recall the relations

$$
x \circ y=V_{x, y} 1=V_{x, 1} y=V_{1, x} y=U_{x, 1} y=U_{x, y} 1 .
$$


2.2. Linear Jordan algebras. If $2 \in k$ is a unit, with inverse $\frac{1}{2} \in k$, we introduce the (bilinear) Jordan product

$$
x y:=x \cdot y:=\frac{1}{2} x \circ y,
$$

making $J$ a linear Jordan algebra in the sense that it is a unital commutative non-associative $k$-algebra with unit $1=1_{J}$ satisfying the Jordan identity

$$
x\left(x^{2} y\right)=x^{2}(x y) .
$$

The $U$-operator of $J$ can be recovered from the Jordan product via the formula $U_{x} y=2 x(x y)-x^{2} y$. Using this, one has no difficulty in setting up a categorical isomorphism between Jordan algebras and linear Jordan algebras over rings containing $\frac{1}{2}$.

2.3. Powers. Returning to the setting of an arbitrary base ring, powers of an element $x \in J$ with integer exponents $\geq 0$ are defined inductively by

$$
x^{0}:=1, \quad x^{1}:=x, \quad x^{n+2}=U_{x} x^{n} .
$$

The submodule of $J$ spanned by the powers of $x$ will be written as

$$
k[x]:=\sum_{n \geq 0} k x^{n} .
$$

It comes equipped with the surjective linear evaluation map

$$
k[\mathbf{t}] \longrightarrow k[x], \quad f \longmapsto f(x),
$$

where $\mathbf{t}$ is a variable. However, even though powers in Jordan algebras are quite well behaved, e.g., by satisfying the relations $U_{x^{m}} x^{n}=x^{2 m+n},\left\{x^{m} x^{n} x^{p}\right\}=2 x^{m+n+p}$, hence forcing $k[x] \subseteq J$ to be a Jordan subalgebra, this subalgebra will in general not be a linear associative $k$-algebra in its own right, making the evaluation map an algebra homomorphism [6, 1.31-32]. On the other hand, by [6, 1.26, QJ37], we do have the formula $U_{(f g)(x)}=U_{f(x)} U_{g(x)}$ for all $f, g \in k[\mathbf{t}]$, which implies that

$$
\text { the linear operators } U_{a}, U_{b} \text { commute for all } a, b \in k[x] \text {. }
$$

Things become much simpler when dealing with linear Jordan algebras $J$ (over rings containing $\frac{1}{2}$ ), because they are power associative, so $k[x] \subseteq J$ is always a unital commutative associative subalgebra.

2.4. Inverses and Jordan division algebras. An element $x \in J$ is said to be invertible (in $J$ ) if there exists an element $y \in J$, necessarily unique, such that $U_{x} y=x$ and $U_{x} y^{2}=1$. We call $x^{-1}:=y$ the inverse of $x$ (in $J$ ) and know from [7, Prop. 1.6.2] that $x$ is invertible iff the linear map $U_{x}: J \rightarrow J$ is bijective iff 1 belongs to the range of $U_{x}$, in which case $x^{-1}=U_{x}^{-1} x$. We call $J$ a Jordan division algebra if $J \neq\{0\}$ and all its non-zero elements are invertible.

2.5. Special versus exceptional Jordan algebras. Let $A$ be a unital associative algebra over $k$. Then the $k$-module $A$ together with the unit $1:=1_{A}$ and the $U$ operator

$$
U_{x} y:=x y x \quad(x, y \in A)
$$


is a Jordan algebra, said to be associated with $A$ and denoted by $A^{+}$. Its Jordan triple product and its circle product are respectively given by

$$
\{x y z\}=x y z+z y x, \quad x \circ y=x y+y x \quad(x, y, z \in A) .
$$

Powers in $A$ and $A^{+}$coincide, as do inverses, so $A^{+}$is a Jordan division algebra iff $A$ is an associative one. A Jordan algebra is said to be special if it is isomorphic to a subalgebra of $A^{+}$, for some unital associative algebra $A$. Typical examples of special Jordan algebras have the form

$$
\operatorname{Her}(A, \tau):=\{x \in A \mid \tau(x)=x\},
$$

$A$ being as above and $\tau$ being an involution of $A$, i.e., an anti-automorphism of period 2. Jordan algebras which are not special are called exceptional. The most important examples of exceptional Jordan algebras are Albert algebras. They will be discussed in more detail in Section 4 below.

2.6. Identities in Jordan algebras. Jordan algebras satisfy a host of useful identities, some of them compiled in [6,7]. In the present paper, the following ones will be needed:

(i) $V_{a} U_{b}=U_{a \circ b, b}-U_{b} V_{a}$,

(ii) $2 U_{a}=V_{a}^{2}-V_{a^{2}}$,

(iii) $a^{2} \circ b^{2}=\{a, a \circ b, b\}-a \circ U_{b} a$,

(iv) $a \circ U_{b} a=b \circ U_{a} b$,

(v) $U_{a} b^{2}=-(a \circ b)^{2}+U_{b} a^{2}+\{a, b, a \circ b\}$,

(vi) $U_{a} U_{b}+U_{b} U_{a}-U_{a \circ b}=U_{U_{a} b, b}-V_{a} U_{b} V_{a}$,

(vii) $V_{a^{2}, b}=V_{a, a \circ b}-V_{U_{a} b}, V_{b, a^{2}}=V_{b \circ a, a}-V_{U_{a} b}$,

(viii) $V_{a} V_{b, a}=V_{U_{a} b}+V_{b} U_{a}$,

(ix) $V_{a} U_{a}=U_{a} V_{a}$,

(x) $V_{a^{2}} U_{a}=U_{a} V_{a^{2}}$.

Rather than pointing out a specific reference for the above identities (some of them are even part of the very definition of a Jordan algebra), we invoke Macdonald's Principle [9, p. 686] and simply note that they hold in special Jordan algebras, hence are valid in general since they either involve less than three variables or exactly three but are linear in one of them.

\section{Motivation: The Connection with Moufang Sets}

In this section, we give a brief survey of how question (111) enters into the connection between Moufang sets and Jordan division rings. Referring to De MedtsSegev [1] and De Medts-Weiss [2] for more details, we recall that a Moufang set is a pair $\mathbb{M}=(X, \mathfrak{S})$ consisting of a set $X$ with more than two elements and a family $\mathfrak{S}=\left(S_{x}\right)_{x \in X}$ of subgroups of $\operatorname{Sym}(X)$, the full permutation group of $X$, such that, writing $G=G_{\mathbb{M}}$ for the subgroup of $\operatorname{Sym}(X)$ generated by the $S_{x}$ 's ("the little projective group of $\mathbb{M}$ "), the following conditions hold: (i) The $S_{x}$ 's make up a full conjugacy class of subgroups of $G$, (ii) for each $x \in X, S_{x}$ is a normal subgroup of $G_{x}$ (the stabilizer of $x$ in $G$ ) and is simply transitive on $X \backslash\{x\}$. M.

For a Moufang set $\mathbb{M}=(X, \mathfrak{S})$ as above, the $S_{x}$ 's are called the root groups of

Now suppose $J$ is a Jordan division ring and, with a new symbol $\infty$, put $X:=$ $J \cup\{\infty\}$. We partially extend the algebraic operations of $J$ to all of $X$ via $a+\infty=$ $\infty=\infty+a(a \in J),-\infty=\infty, \infty^{-1}=0,0^{-1}=\infty$ and use this to define 
permutations of $X$ by means of $\alpha_{a}: X \rightarrow X, x \mapsto a+x(a \in J), \tau: X \rightarrow X$, $x \mapsto-x^{-1}$. It then follows that $\mathbb{M}(J):=(X, \mathfrak{S}), \mathfrak{S}=\left(S_{x}\right)_{x \in X}$ with

$$
S_{\infty}:=\left\{\alpha_{a} \mid a \in J\right\}, \quad S_{0}=\tau S_{\infty} \tau^{-1}, \quad S_{a}:=\alpha_{a} S_{0} \alpha_{a}^{-1} \quad\left(a \in J^{\times}\right)
$$

is a Moufang set with root groups all isomorphic to the additive group of $J$; in particular, they are abelian.

An important question in the theory of Moufang sets is the converse: does every Moufang set with abelian root groups come from a Jordan division ring? Since this question in its general form looks rather intractable at the moment, Segev has suggested that one consider special cases, like the one defined by the Zassenhaus condition: a Moufang set $\mathbb{M}=(X, \mathfrak{S})$ is said to satisfy the Zassenhaus condition if $G:=G_{\mathbb{M}}$ is not sharply 2-transitive on $X$ and the pointwise stabilizer in $G$ of three distinct points of $X$ is trivial.

This concept gives rise to the following natural question: which Jordan division rings have the property that their associated Moufang sets satisfy the Zassenhaus condition? The following answer, due to Segev and Tent (unpublished), highlights the significance of question (111) in the present context: If $J$ is a Jordan division ring of characteristic not 2 such that $\mathbb{M}(J)$ satisfies the Zassenhaus condition, and (11) can be answered affirmatively for $J$, then $J$ is a commutative field; more precisely, there exists a field $F$ such that $J=F^{+}$.

\section{THE ORIGINAL QUESTION}

In this section we will address question (111) in its original set-up of a Jordan division ring. We begin by disposing of a trivial but crucial side issue.

4.1. The case of a special Jordan algebra. Let $J$ be a special Jordan algebra over $k$, so there is a unital associative $k$-algebra $A$ such that $J$ is a subalgebra of $A^{+}$. Hence the $U$-operator and the circle product of $J$ are given by (2.511) and the second equation of (2.512) in terms of the associative product of $A$. Now suppose $x, y \in J$ satisfy the relation $x \circ y=0$. Then $x y=-y x$ and the expression $U_{x} U_{y} z=x y z y x$ is symmetric in $x$ and $y$. Thus question (111) has an affirmative answer for special Jordan algebras.

In order to proceed, we require a comparatively short digression into Albert algebras, which we will treat here in a slightly unusual way, by focussing exclusively on the objectives of the present paper. For simplicity, we therefore replace our commutative base ring $k$ by an arbitrary field $F$. Free use will then be made of the differential calculus for polynomial maps as explained in Jacobson [5. Chap. VI], with a few notational adjustments taken from McCrimmon [10.

4.2. Cubic norm structures. Combining the conceptual framework of McCrimmon [10] with the terminology of Petersson-Racine [18, we define a cubic norm structure over $F$ as a quadruple $X=(X, 1, N, \sharp)$ consisting of a vector space $X$ over $F$, a distinguished element $1 \in X$ (the base point), a cubic form $N: X \rightarrow F$ (the norm) and a quadratic map $X \rightarrow X, x \mapsto x^{\sharp}$ (the adjoint) such that $N(1)=1$, $1^{\sharp}=1$ and the following identities hold under all scalar extensions:

$$
\begin{aligned}
1 \times x & =T(x) 1-x, \\
T\left(x^{\sharp}, y\right) & =\left(\partial_{y} N\right)(x), \\
x^{\sharp \sharp} & =N(x) x .
\end{aligned}
$$


Here $x \times y=(x+y)^{\sharp}-x^{\sharp}-y^{\sharp}$ is the bilinearized adjoint, $T: X \times X \rightarrow F$ defined by

$$
T(x, y)=\left(\partial_{x} N\right)(1)\left(\partial_{y} N\right)(1)-\left(\partial_{x} \partial_{y} N\right)(1)
$$

is the bilinear trace of $X$, and $T(x)=T(x, 1)$. We also put $S(x):=T\left(x^{\sharp}\right)$ and call the map $T: X \rightarrow k$ (resp. $S: X \rightarrow k$ ) the linear (resp. the quadratic) trace of $X$, the latter being a quadratic form whose polarization satisfies the relation

$$
S(x, y)=T(x) T(y)-T(x, y) .
$$

The $F$-vector space $X$ carries canonically a Jordan algebra structure $J=J(X)$ whose unit agrees with the base point of $X$ and whose $U$-operator is given by

$$
U_{x} y=T(x, y) x-x^{\sharp} \times y .
$$

$J$ is a cubic Jordan algebra over $F$ in the sense that the relation

$$
x^{3}-T(x) x^{2}+S(x) x-N(x) 1=0
$$

holds in all scalar extensions. Moreover, we have

$$
\begin{aligned}
x^{\sharp} & =x^{2}-T(x) x+T\left(x^{\sharp}\right) 1, \\
x \times y & =x \circ y-T(x) y-T(y) x+(T(x) T(y)-T(x, y)) 1 .
\end{aligned}
$$

From (3) and (15) we deduce that any automorphism of $X$, i.e., any linear bijection $X \rightarrow X$ preserving unit, norm and adjoint, is an automorphism of $J$.

4.3. Alternative algebras of degree 3. Let $A$ be a unital algebra over $F$ which is alternative in the sense that the associator $[x, y, z]:=(x y) z-x(y z)$ is alternating, equivalently, that any subalgebra on two generators is associative. Then the $F$ vector space $A$ together with the unit $1=1_{A}$ and the $U$-operator $U_{x} y:=(x y) x=$ $x(y x)=: x y x$ is a Jordan algebra, actually a special one, said to be associated with $A$ and denoted by $A^{+}$.

Now suppose $A$ is a finite-dimensional alternative $F$-algebra of degree 3 in the sense of [5, p. 223] and write $N:=N_{A}$ for its (generic) norm. By [5, Theorems VI.1,VI.3] (see also Faulkner [4, Lemma]), the relations

$$
N(1)=1, \quad N(x y)=N(x) N(y)
$$

hold in all scalar extensions. Defining

$$
\begin{aligned}
T(x, y) & =\left(\partial_{x} N\right)(1)\left(\partial_{y} N\right)(1)-\left(\partial_{x} \partial_{y} N\right)(1), \\
T(x) & =T(x, 1), \\
x^{\sharp} & =x^{2}-T(x) x+\left(\partial_{1} N\right)(x) 1,
\end{aligned}
$$

as in Section 4.2, one checks that $X:=X(A):=(A, 1, N, \sharp)$ is a cubic norm structure over $F$ with bilinear trace $T(x, y)=T(x y)$ and associated cubic Jordan algebra $J(X)=A^{+}$. Moreover, $T$ is also the generic trace of $A$, and for $u, v \in A$, we have the additional relation

$$
(u v)^{\sharp}=v^{\sharp} u^{\sharp},
$$


which (repeatedly) linearizes to

$$
\begin{aligned}
\left(u_{1} v\right) \times\left(u_{2} v\right) & =v^{\sharp}\left(u_{1} \times u_{2}\right), \\
\left(u v_{1}\right) \times\left(u v_{2}\right) & =\left(v_{1} \times v_{2}\right) u^{\sharp}, \\
\left(u_{1} v_{1}\right) \times\left(u_{2} v_{2}\right)+\left(u_{1} v_{2}\right) \times\left(u_{2} v_{1}\right) & =\left(v_{1} \times v_{2}\right)\left(u_{1} \times u_{2}\right) .
\end{aligned}
$$

Most alternative algebras of degree 3 we encounter in the sequel will be separable in the sense that they stay semi-simple under all base field extensions, equivalently, that their bilinear trace is a non-degenerate symmetric bilinear form.

4.4. The first Tits construction. Let $A$ be a finite-dimensional alternative $F$ algebra of degree 3, with generic norm $N$, generic trace $T$ as in Section 4.3, and $\mu \in F$. Following McCrimmon [10, Theorem 6] if $A$ is associative and Faulkner [4] or Petersson-Racine [18 in the general case, the direct sum of three copies of $A$ as an $F$-vector space, written as

$$
X:=A \oplus A j_{1} \oplus A j_{2},
$$

becomes a cubic norm structure $X(A, \mu)$ over $F$ whose base point, norm, (bilinearized) adjoint, and trace are extended from $X(A)$ by means of the formulas

$$
\begin{aligned}
1= & 1+0 j_{1}+0 j_{2}, \\
N(x)= & N\left(x_{0}\right)+\mu N\left(x_{1}\right)+\mu^{2} N\left(x_{2}\right)-\mu T\left(x_{0} x_{1} x_{2}\right), \\
x^{\sharp}= & \left(x_{0}^{\sharp}-\mu x_{1} x_{2}\right)+\left(\mu x_{2}^{\sharp}-x_{0} x_{1}\right) j_{1}+\left(x_{1}^{\sharp}-x_{2} x_{0}\right) j_{2}, \\
x \times y= & \left(x_{0} \times y_{0}-\mu x_{1} y_{2}-\mu y_{1} x_{2}\right)+\left(\mu x_{2} \times y_{2}-x_{0} y_{1}-y_{0} x_{1}\right) j_{1} \\
& +\left(x_{1} \times y_{1}-x_{2} y_{0}-y_{2} x_{0}\right) j_{2}, \\
T(x, y)= & T\left(x_{0}, y_{0}\right)+\mu T\left(x_{1}, y_{2}\right)+\mu T\left(x_{2}, y_{1}\right), \\
T(x)= & T\left(x_{0}\right)
\end{aligned}
$$

for

$$
x=x_{0}+x_{1} j_{1}+x_{2} j_{2}, \quad y=y_{0}+y_{1} j_{1}+y_{2} j_{2} \quad\left(x_{i}, y_{i} \in A, i=0,1,2\right) .
$$

The cubic Jordan algebra corresponding to $X(A, \mu)$ will be denoted by $J(A, \mu)$. Both are said to arise from $A, \mu$ by means of the first Tits construction; there is also a second construction, but we won't need it here. Note that $A^{+}=J(X(A))$ embeds into $J(A, \mu)$ as a subalgebra through the initial summand.

4.5. The concept of an Albert algebra. The algebra $\operatorname{Mat}_{3}(F)$ of ordinary $3 \times 3$ matrices over $F$ is central simple associative of degree 3 , with generic norm (resp. generic trace) given by the ordinary determinant (resp. trace) of matrices, while their usual adjoint agrees with the adjoint of the corresponding cubic norm structure.

This being so, we call $J_{\mathrm{spl}}:=J\left(\operatorname{Mat}_{3}(F), 1\right)$, i.e., the cubic Jordan algebra arising from $A=\operatorname{Mat}_{3}(F)$ and $\mu=1$ by means of the first Tits construction, the split Albert algebra over $F$. By an Albert algebra over $F$, we mean a Jordan $F$-algebra which is an $F$-form of $J_{\text {spl }}$, i.e., which becomes isomorphic to $J_{\text {spl }}$ after extending scalars to the separable closure of $F$. Albert algebras are central simple exceptional Jordan algebras of dimension 27 over $F$. By Galois descent, they inherit unit, norm, adjoint and trace from $J_{\text {spl }}$. Typical examples of Albert algebras are first Tits constructions $J(A, \mu)$, where $A$ is any central simple associative algebra of degree 3 and $\mu \in F$ is a non-zero scalar. Conversely, suppose $J$ is an Albert algebra 
and $A$ is a central simple associative algebra of degree 3. By [11, Theorem 8] and its proof, any embedding $A^{+} \hookrightarrow J$ of Jordan algebras can be extended to an isomorphism $J(A, \mu) \stackrel{\sim}{\rightarrow} J$, for some non-zero scalar $\mu \in F$.

4.6. Albert division algebras. Albert division algebras, i.e., Albert algebras that are Jordan division algebras in the sense of Section 2.4 exist and are easy to construct. For example, given an associative $F$-algebra $A$ of degree 3 and a nonzero scalar $\mu \in F$, the first Tits construction $J(A, \mu)$ is an Albert division algebra iff $\mu$ is not a generic norm of $A$, in which case $A$ will be an associative division algebra. Moreover, an Albert algebra is division iff its norm is anisotropic. Hence, by the theorem of Chevalley-Warning [8, Exercise IV.7], there are no Albert division algebras over finite fields and, by a theorem of Springer [19, Lemma 4.2.11], the property of being an Albert division algebra is preserved under quadratic field extensions.

4.7. Subalgebras on two generators. It is a standard fact that subalgebras on two generators of an Albert division algebra exist only in dimensions 1,3, 9. Since we are not aware of a convenient reference in arbitrary characteristic, we briefly sketch an ad-hoc proof. Let $J$ be an Albert division algebra over $F$, with unit 1 , norm $N$, adjoint $\sharp$, trace $T$, and $J^{\prime} \subseteq J$ a subalgebra on two generators, which we may assume to have dimension $>1$. Then $F$ is infinite (4.6), and for $\operatorname{char}(F) \neq 2$, the ShirshovCohn theorem [5, Theorem I.10] ensures that $J^{\prime}$ is special, which, when combined with [21, p. 148] and the exceptional character of Albert algebras (4.5), yields the assertion. For the remainder of the proof, we may therefore assume $\operatorname{char}(F)=2$ (in fact, assuming $\operatorname{char}(F) \neq 3$ will do). Then the validity of the Shirshov-Cohn theorem is no longer guaranteed but, by a Zariski density argument, we find two generators $a, b$ of $J^{\prime}$ such that $a$ generates a separable cubic subfield $E \subseteq J^{\prime}$. If $b \in E$, then $J^{\prime}=E$ has dimension 3 , and if $b \notin E$, then $J^{\prime}$ has dimension 9 [21, (30.17)].

We are now in a position to provide an affirmative answer to question (11) in its original form.

Theorem 4.8. Suppose we are given elements $x, y$ in a Jordan division ring satisfying $x \circ y=0$. Then the $U$-operators $U_{x}$ and $U_{y}$ commute: $U_{x} U_{y}=U_{y} U_{x}$.

Proof. Write $J$ for the Jordan division ring in question. By the McCrimmonZel'manov structure theorem [15, 15.7], $J$ is either special or an Albert division algebra over some field $F$. The former case having been settled in Section 4.1, we may assume the latter. Since $J$ has degree 3 over $F$, it follows from [17, Cor. 3] that non-zero elements $x, y \in J$ having $x \circ y=0$ do not exist unless $F$ has characteristic 2. Thus, in view of (2.311), the proof of Theorem 4.8 will be complete once we have shown the following proposition.

Proposition 4.9. Let $J$ be an Albert division algebra over a field $F$ of characteristic 2 and suppose $x, y \in J$ satisfy $x \circ y=0$. Then $x \in F 1$ or $y \in F[x]$.

Proof. Assume the contrary, so $x \notin F 1$ and $y \notin F[x]$. Then $J^{\prime}$, the subalgebra of $J$ generated by $x, y$, has dimension 9 (4.7). Hence, by [21, p. 148], $J^{\prime}$ is either (i) a purely inseparable field extension of characteristic 3 and exponent at most 1 over $F$, (ii) of the form $D^{+}$for some central associative division algebra $D$ of degree 3 over $F$, or (iii) of the form $\operatorname{Her}(D, \tau)$, the Jordan algebra of $\tau$-symmetric elements in a central associative division algebra $(D, \tau)$ of degree 3 with involution 
of the second kind over $F$. Here (i) is impossible since $F$ has characteristic 2 , and (iii) will be converted into (ii) after extending scalars to an appropriate separable quadratic field extension. We may therefore assume $J^{\prime}=D^{+}$as in (ii). Since $F$ has characteristic 2 , the relation $x \circ y=0$ implies that $x$ and $y$ commute in $D$. But $k[x]$, being a separable cubic subfield of $D$, agrees with its own centralizer, which implies $y \in k[x]$, a contradiction.

Remark. Roughly speaking, the reasons for the validity of Theorem 4.8 are (i) the result is trivial for special Jordan algebras, (ii) elements $x, y$ in Albert division algebras satisfying $x \circ y=0$ are extremely rare. The ubiquity of such elements in Jordan algebras that fail to be division is well documented in the special cases below and seems to be responsible for the intractability of the problem in its most general form.

\section{A first approach to the General CASE.}

In this section, we fix an arbitrary Jordan algebra $J$ over $k$ and derive a number of consequences of the relation $x \circ y=0$ that turn out to be useful in studying question (11) in its most general form.

Proposition 5.1. Assume that $x, y \in J$ satisfy $x \circ y=0$. Then:

(i) $x^{n} \circ y=0$ for all odd integers $n>0$,

(ii) $V_{x} U_{y}=-U_{y} V_{x}$,

(iii) $x^{2} \circ y=-2 U_{x} y$,

(iv) $U_{y} x^{2}=U_{x} y^{2}$

(v) $x^{2} \circ y^{2}=-x \circ U_{y} x=2 U_{y} x^{2}$,

(vi) $U_{x} U_{y}-U_{y} U_{x}=U_{U_{x} y, y}+U_{y} V_{x^{2}}=-U_{U_{x} y, y}-V_{x^{2}} U_{y}$,

(vii) $V_{x^{2}, y}=-V_{U_{x} y}=V_{y, x^{2}}$.

The identities obtained from (i)-(vii) by interchanging $x$ and $y$ also hold. For further reference we will indicate them with an asterisk as a superscript.

Proof. (i) We proceed by induction on $n$. The assertion is true for $n=1$. Given any odd integer $n \geq 3$, let us assume that $x^{n-2} \circ y=0$. Then $y \circ x^{n}=V_{y} U_{x} x^{n-2}=$ $U_{y \circ x, x} x^{n-2}-U_{x}\left(y \circ x^{n-2}\right)$ (by Section 2.6 (i) $)=0$ by assumption and the induction hypothesis.

(ii) Apply Section 2.6(i) with $a=x$ and $b=y$.

(iii) Apply Section 2.6(ii) with $a=x$ acting on $y$.

(iv) Apply Section [2.6(v) with $a=x$ and $b=y$.

(v) Apply Section 2.6(iii) with $a=x$ and $b=y$ to obtain the first equality, and, for the second one, use (ii).

(vi) $U_{x} U_{y}+U_{y} U_{x}=U_{U_{x} y, y}-V_{x} U_{y} V_{x}$ (by Section 2.6(vi)) $=U_{U_{x} y, y}+U_{y} V_{x}^{2}$ (by (ii)) $=U_{U_{x} y, y}+2 U_{y} U_{x}+U_{y} V_{x^{2}}$ (by Section 2.6 (ii)), which readily implies the first equality.

Again $U_{x} U_{y}+U_{y} U_{x}=U_{U_{x} y, y}-V_{x} U_{y} V_{x}$ (by Section 2.6(vi)) $=U_{U_{x} y, y}+V_{x}^{2} U_{y}$ (by (ii)) $=U_{U_{x} y, y}+2 U_{x} U_{y}+V_{x^{2}} U_{y}$ (by Section 2.6(ii)), which implies $U_{x} U_{y}-U_{y} U_{x}=$ $-U_{U_{x} y, y}-V_{x^{2}} U_{y}$.

(vii) Apply Section 2.6(vii) with $a=x$ and $b=y$.

The final assertion is obvious. 
Proposition 5.2. Assume that $x, y \in J$ satisfy $x \circ y=U_{x} y=0$. Then:

(i) $x^{n} \circ y=0$ for all positive integers $n$,

(ii) $x^{2} \circ y^{2}=2 U_{x} y^{2}=2 U_{y} x^{2}=x \circ U_{y} x=0$,

(iii) $V_{x^{2}, y}=V_{y, x^{2}}=0$,

(iv) $2 V_{x^{2}} U_{y}=2 U_{y} V_{x^{2}}=0$,

(v) $2 U_{x} U_{y}=2 U_{y} U_{x}$.

Proof. (i) We have $x^{2} \circ y=0$ as a consequence of Proposition 5.1(iii). Now, for any $n \geq 3, x^{n}=U_{x} x^{n-2}$ and we can prove the assertion by induction on $n$ as in Proposition 5.1(i).

(ii) follows directly from Proposition $5.1(\mathrm{v})$ and Proposition $5.1(\mathrm{v})^{*}$.

(iii) follows directly from Proposition 5.1(vii).

(iv) Using (iii),

$0=2 V_{y} V_{x^{2}, y}=2 V_{U_{y} x^{2}}+2 V_{x^{2}} U_{y}$ (by Section 2.6 (viii) with $a=y$ and $b=x^{2}$ )

$=V_{2 U_{y} x^{2}}+2 V_{x^{2}} U_{y}=2 V_{x^{2}} U_{y}$

by (ii). Now $2 U_{y} V_{x^{2}}=-2 V_{x^{2}} U_{y}=0$ by Proposition 5.1(ii) applied to $x^{2}$ and $y$ since $x^{2} \circ y=0$ by (i).

(v) Just notice that $2\left(U_{x} U_{y}-U_{y} U_{x}\right)=0$ by using (iv) and Proposition 5.1(vi).

Proposition 5.3. Assume that $x, y \in J$ satisfy $x \circ y=0$. If $x$ is invertible, then $x^{-1} \circ y=0$.

Proof. $U_{x}\left(x^{-1} \circ y\right)=U_{x} V_{y}\left(x^{-1}\right)=-V_{y} U_{x}\left(x^{-1}\right)$ (by Proposition 5.1 (ii) $\left.{ }^{*}\right)=-V_{y} x=$ $-y \circ x=0$, hence $x^{-1} \circ y=0$ since $U_{x}$ is invertible.

\section{Algebraic elements of LOW Degree in linear Jordan Algebras}

In this section, we fix elements $x, y$ in a linear Jordan algebra $J$ over a field $F$ of characteristic not 2 (cf. Section 2.2). Our aim is to answer question (111) affirmatively in the presence of certain algebraicity conditions. Referring to [5, VI.3] for details, let us begin by recalling some basic concepts.

6.1. Algebraic elements. $x$ is said to be algebraic if the unital commutative associative subalgebra $F[x]$ of $J$ is finite-dimensional over $F$. In this case, the minimal polynomial of $x$ in a variable $\mathbf{t}$, denoted by $\mu_{x}(\mathbf{t}) \in F[\mathbf{t}]$, can be formed with respect to this subalgebra and has the usual properties. For example, $x$ is invertible in $J$ iff it is so in $F[x]$ iff $\mu_{x}(0) \neq 0$, in which case $x^{-1} \in F[x]$. At the other extreme, $x$ is nilpotent iff it is algebraic with $\mu_{x}(\mathbf{t})=\mathbf{t}^{n}$ for some positive integer $n$. The degree of an algebraic element in $J$ is defined as the degree of its minimal polynomial.

Proposition 6.2. If $x$ is algebraic of degree at most 2 and $x \circ y=0$, then $U_{x} U_{y}=$ $U_{y} U_{x}$.

Proof. If $x$ is algebraic of degree 1 , then it is a scalar multiple of 1 , and $U_{x} U_{y}=$ $U_{y} U_{x}$, for any $y \in J$. Hence we may assume that $x$ is algebraic of degree 2 , forcing $\mu_{x}(\mathbf{t})=\mathbf{t}^{2}+\alpha \mathbf{t}+\beta$ for some $\alpha, \beta \in F$ and, in particular, $x^{2}+\alpha x+\beta 1=0$. If $\beta \neq 0$, then $x$ is invertible by Section 6.1 with inverse $x^{-1}=-\beta^{-1}(x+\alpha 1)$. Thus, by Proposition 5.3 .

$$
0=x^{-1} \circ y=-\beta^{-1}(x+\alpha 1) \circ y=-2 \beta^{-1} \alpha y,
$$


so either $y=0$, which obviously implies the assertion, or $\alpha=0$. In the latter case, $x^{2}=-\beta 1, V_{x^{2}}=-2 \beta \operatorname{Id}_{J} ;$ thus

$$
V_{x^{2}} U_{y}=U_{y} V_{x^{2}}
$$

and

$$
\begin{aligned}
2 U_{x} U_{y} & =\left(V_{x}^{2}-V_{x^{2}}\right) U_{y}(\text { by Section 2.6 (ii)) } \\
& \left.=U_{y}\left(V_{x}^{2}-V_{x^{2}}\right) \text { (by Proposition 5.1(ii) and (10) }\right)=2 U_{y} U_{x} .
\end{aligned}
$$

If $\beta=0$, then $x^{2}=-\alpha x$ and $x^{2} \circ y=-\alpha x \circ y=0$. Hence $2 U_{x} y=0$ by Proposition 5.1(iii), which yields $U_{x} y=0$ since the characteristic is not two. Thus $2 U_{x} U_{y}=2 U_{y} U_{x}$ by Proposition $\left[5.2(\mathrm{v})\right.$, and $U_{x} U_{y}=U_{y} U_{x}$, again using the fact that the characteristic is not two.

Proposition 6.3. If $x$ is algebraic of degree 3 and $x, y$ are both invertible, then $x \circ y \neq 0$.

Proof. Arguing indirectly, let us assume $x \circ y=0$. By Section 6.1 we have $\mu_{x}(\mathbf{t})=$ $\mathbf{t}^{3}+\alpha \mathbf{t}^{2}+\beta \mathbf{t}+\gamma$ for some $\alpha, \beta, \gamma \in F, \gamma \neq 0$. Thus $x^{-1}=-\gamma^{-1}\left(x^{2}+\alpha x+\beta 1\right)$, which implies that $x^{2}$ is a linear combination of $x^{-1}, x$, and 1 . Therefore, using Proposition 5.3, $x^{2} \circ y$ is a scalar multiple of $y$ and the same holds true for $U_{x} y$ by Proposition 5.1(iii). Let us say that

$$
U_{x} y=\delta y
$$

for some $\delta \in F$. We have

$$
\begin{aligned}
U_{x} y^{2}=\frac{1}{2} U_{x} V_{y} y & =-\frac{1}{2} V_{y} U_{x} y(\text { by Proposition [5.1(ii)*) } \\
& =-\frac{1}{2} y \circ(\delta y)(\text { by (1) })=-\delta y^{2} .
\end{aligned}
$$

Thus

$$
U_{y} x^{2}=U_{x} y^{2}\left(\text { by Proposition 5.1(iv)) }=-\delta y^{2}(\text { by (2) })=U_{y}(-\delta 1),\right.
$$

i.e., $U_{y}\left(x^{2}+\delta 1\right)=0$. But $y$ is invertible by hypothesis, forcing $x^{2}+\delta 1=0$, so $x$ is algebraic of degree at most two, a contradiction.

Remark. The preceding result generalizes [17, Cor. 3], which says that in cubic Jordan division algebras of characteristic not 2 non-zero elements $x, y$ with $x \circ y=0$ do not exist.

Proposition 6.4. If $x$ is algebraic of degree 3 and neither invertible nor nilpotent, then $x \circ y=0$ implies $U_{x} U_{y}=U_{y} U_{x}$.

Proof. This time Section 6.1 yields $\mu_{x}(\mathbf{t})=\mathbf{t}^{3}+\alpha \mathbf{t}^{2}+\beta \mathbf{t}$ for some $\alpha, \beta \in F$ not both zero. If $\alpha \neq 0$, then $x^{2}$ is a linear combination of $x^{3}$ and $x$, hence $x^{2} \circ y=0$ by Proposition 5.1(i), $U_{x} y=0$ by Proposition 5.1(iii), and $U_{x} U_{y}=U_{y} U_{x}$ by Proposition $5.2(\mathrm{v})$.

Hence we may assume $\alpha=0$, forcing $x^{3}=-\beta x, \beta \neq 0$. After extending scalars to the algebraic closure of $F$, we may replace $x$ by $(-\beta)^{-\frac{1}{2}} x$ in order to ensure $x^{3}=x$.

Now $U_{x}^{3}=U_{x^{3}}=U_{x}$ implies that the minimal polynomial of the endomorphism $U_{x}: J \longrightarrow J$ divides $X^{3}-X=(X-1)(X+1) X$. Hence $U_{x}$ is diagonalizable and 
$J=J_{0} \oplus J_{1} \oplus J_{-1}$, where $J_{\varepsilon}=\left\{z \in J \mid U_{x} z=\varepsilon z\right\}, \varepsilon \in\{0,1,-1\}$. The fact that $U_{x}$ and $V_{x}$ commute by Section 2.6(ix) implies

$$
V_{x}\left(J_{\varepsilon}\right) \subseteq J_{\varepsilon}
$$

$\varepsilon \in\{0,1,-1\}$ (if $z \in J_{\varepsilon}, U_{x} V_{x} z=V_{x} U_{x} z=V_{x}(\varepsilon z)=\varepsilon V_{x} z$ ). Similarly, using Section 2.6 (x), we obtain

$$
V_{x^{2}}\left(J_{\varepsilon}\right) \subseteq J_{\varepsilon} .
$$

We can write $y=y_{0}+y_{1}+y_{-1}$, with $y_{\varepsilon} \in J_{\varepsilon}, \varepsilon \in\{0,1,-1\}$, and $V_{x}(y)=0$ together with (10) yields

$$
V_{x}\left(y_{\varepsilon}\right)=x \circ y_{\varepsilon}=0,
$$

$\varepsilon \in\{0,1,-1\}$. On the other hand, $e:=x^{2}$ is an idempotent $\left(e^{2}=x^{4}=\frac{1}{2} x^{3} \circ x=\right.$ $\frac{1}{2} x \circ x=x^{2}=e$ ) and $e \circ y_{1}=x^{2} \circ y_{1}=-2 U_{x} y_{1}$ (by (3) and Proposition 5.1(iii)) $=-2 y_{1}$, which implies

$$
y_{1}=0, \quad y=y_{0}+y_{-1},
$$

since the only possible eigenvalues of $V_{e}$ are $0,1,2$ [14, II.8.1.4] because $e$ is an idempotent.

Let $J=J_{0}(e) \oplus J_{1}(e) \oplus J_{2}(e)$ be the Peirce decomposition of $J$ with respect to $e$ [14, II.8.1.2(1)]. In what follows, free use will be made of the rules governing multiplication of the Peirce components [14, II, 8.2.1].

Since $U_{e} J_{0}=U_{x^{2}} J_{0}=U_{x}^{2} J_{0}=0$, we have $J_{0} \subseteq J_{0}(e) \oplus J_{1}(e)$, while for any $z \in J_{i}, i= \pm 1, U_{e} z=U_{x^{2}} z=U_{x}^{2} z=i^{2} z=z$, which implies $J_{1} \oplus J_{-1} \subseteq J_{2}(e)$. Thus, we have

$$
J_{0}=J_{0}(e) \oplus J_{1}(e), \quad J_{1} \oplus J_{-1}=J_{2}(e) .
$$

For $y_{0}$ we can be more precise since $e \circ y_{0}=x^{2} \circ y_{0}=-2 U_{x} y_{0}$ (by Proposition 5.1 (iii) using (3) $)=0$, so that

$$
y_{0} \in J_{0}(e) .
$$

We recall a fact that will be needed later:

$$
U_{x} U_{y_{0}}=U_{y_{0}} U_{x}
$$

which follows directly from (3), the fact that $U_{x} y_{0}=0$, and Proposition $5.2(\mathrm{v})$.

Now we show $U_{x} U_{y}=U_{y} U_{x}$ by checking that both sides coincide when restricted to $J_{0}$ and $J_{2}(e)$.

(I) If $z \in J_{0}$, then

$$
U_{y} z=U_{y_{0}+y_{-1}} z=U_{y_{0}} z+U_{y_{-1}} z+\left\{y_{0} z y_{-1}\right\}=U_{y_{0}} z+\left\{y_{0} z y_{-1}\right\}
$$

since $U_{y_{-1}} z \in U_{J_{2}(e)}\left(J_{0}(e)+J_{1}(e)\right)$ (by (4) $)=0$. Thus

$$
U_{x} U_{y} z=U_{x} U_{y_{0}} z+U_{x}\left\{y_{0} z y_{-1}\right\}=U_{x} U_{y_{0}} z
$$

since $\left\{y_{0}, z, y_{-1}\right\} \in\left\{J_{0}(e), J_{0}(e)+J_{1}(e), J_{2}(e)\right\}$ (by (4) and (5) $) \subseteq J_{1}(e) \subseteq J_{0}$. But by (6), $U_{x} U_{y_{0}} z=U_{y_{0}} U_{x} z=0$, and we have shown $U_{x} U_{y} z=0=U_{y} U_{x} z$.

(II) If $z \in J_{2}(e)$, then

$$
U_{y} z=U_{y_{0}+y_{-1}} z=U_{y_{0}} z+U_{y_{-1}} z+\left\{y_{0} z y_{-1}\right\}=U_{y_{-1}} z
$$

since $U_{y_{0}} z \in U_{J_{0}(e)} J_{2}(e)$ (by (5) $)=0$, and $\left\{y_{0} z y_{-1}\right\} \subseteq\left\{J_{0}(e) J_{2}(e) J\right\}$ (by (5) ) $)=0$. Now $U_{x} z \in J_{2}(e)$ since $x=x^{3}=U_{x} x \in J_{1} \subseteq J_{2}(e)$ by (41). Hence (7) implies $U_{y} U_{x} z=U_{y_{-1}} U_{x} z$, so that $U_{x} U_{y} z=U_{y} U_{x} z$ reduces to $U_{x} U_{y_{-1}} z=U_{y_{-1}} U_{x} z$ which 
holds by Proposition 6.2, $x, z, y_{-1} \in J_{2}(e)$ by (44), $J_{2}(e)$ is a Jordan subalgebra of $J, x \circ y_{-1}=0$ by (3), and $x$ is algebraic of degree two or less in $J_{2}(e)$ since $0=x^{2}-e=x^{2}-1_{J_{2}(e)}$.

\section{PoInted QUADRATIC FORMS}

Since question (111) in its general form has an affirmative answer for special Jordan algebras (see Section 4.1), we have to focus our attention on exceptional ones. While Jordan algebras of pointed quadratic forms are special under mild regularity conditions (see [7, Theorem 2.2.14] for a more precise statement), they are not so in general [7, p. 2.6]. Hence it makes sense to discuss them in the present context.

7.1. Trace and conjugation of a pointed quadratic form. In what follows we fix a pointed quadratic form $(M, q, 1)$ over $k$, so $M$ is a $k$-module, $1 \in M$ is a distinguished element (the base point) and $q: M \rightarrow k$ is a quadratic form (the norm) satisfying $q(1)=1 \in k$. We call $t: M \rightarrow k, x \mapsto q(1, x)$ the trace and $\iota: M \rightarrow M, \quad x \mapsto \bar{x}:=t(x) 1-x$ the conjugation of $(M, q, 1)$. By definition we have

$$
q(1)=1, \quad t(1)=2,
$$

which implies that the conjugation of $(M, q, 1)$ is a linear map of period 2 preserving base point, norm and trace:

$$
\overline{1}=1, \quad q(\bar{x})=q(x), \quad t(\bar{x})=t(x) .
$$

We also have

$$
q(\bar{x}, y)=q(x, \bar{y})=t(x) t(y)-q(x, y) .
$$

7.2. The Jordan algebra of a pointed quadratic form. We now consider the Jordan algebra $J=J(M, q, 1)$ associated with $(M, q, 1)$ [7, 2.1]. Recall, in particular, that $J=M$ as $k$-modules, $1_{J}=1 \in M$ is the unit of $J$, and its $U$-operator acts on $J$ via

$$
U_{x} y=q(x, \bar{y}) x-q(x) \bar{y} .
$$

Linearizing gives $x \circ y=t(x) y+t(y) x-q(x, y) 1$. In particular, the condition $x \circ y=0$ is equivalent to

$$
t(x) y+t(y) x=q(x, y) 1 .
$$

Our aim is to derive a formula for $U_{x} U_{y} z$ (in terms of the norm and its polarization) that is symmetric in $x$ and $y$. We will be able to do so, but, unfortunately, only in the absence of 2-torsion. We begin by applying (7.12) to obtain

$$
\begin{aligned}
q(x) q(\bar{y}, z) \bar{y} & =q(x)(t(y) t(z)-q(y, z))(t(y) 1-y) \\
& =q(x) t(y)^{2} t(z) 1-q(x) t(y) t(z) y-q(x) t(y) q(y, z) 1+q(x) q(y, z) y,
\end{aligned}
$$

after which a straightforward verification yields

$$
\begin{aligned}
U_{x} U_{y} z= & q(x) q(y) z-q(x) q(y, z) y-q(y) q(x, z) x \\
& +q(x, \bar{y}) q(\bar{y}, z) x-q(x) t(y)^{2} t(z) 1+q(x) t(y) q(y, z) 1+q(x) t(y) t(z) y
\end{aligned}
$$

Since the first three terms on the right-hand side of (2) form an expression that is symmetric in $x$ and $y$, it will be enough to show that the remaining ones all belong 
to the 2-torsion part of $J$ provided $x \circ y=0$. This will be accomplished by the following lemma.

Lemma 7.3. If $x, y \in J$ satisfy the relation $x \circ y=0$, then

$$
\begin{aligned}
2 q(x) t(y) & =0=2 t(x) q(y), \\
2 q(x, y) & =2 t(x) t(y), \\
2 q(x, \bar{y}) & =0 .
\end{aligned}
$$

Proof. Given $z \in J$, we invoke (7.21) and obtain $q(x, y) t(z)=q(q(x, y) 1, z)=$ $q(t(x) y+t(y) x, z)$; hence

$$
q(x, y) t(z)=t(x) q(y, z)+t(y) q(x, z) .
$$

Setting $z=x$ in (4) yields $q(x, y) t(x)=t(x) q(x, y)+2 q(x) t(y)$, hence the first relation of (11), which by symmetry implies the second. Setting $z=1$ in (4) and applying (7.111) yields (2), which combines with (7.112) to yield (3).

Theorem 7.4. If $x, y \in J$ satisfy $x \circ y=0$, then $2 U_{x} U_{y}=2 U_{y} U_{x}$; in particular, if there is no 2-torsion, the operators $U_{x}$ and $U_{y}$ commute.

Proof. Combining Lemma 7.3 with (7.22), we conclude that

$$
2 U_{x} U_{y} z=2(q(x) q(y) z-q(x) q(y, z) y-q(y) q(x, z) x)
$$

is symmetric in $x$ and $y$, whence the assertion follows.

Remark. In the absence of 2-torsion, (7.411) yields the formula

$$
U_{x} U_{y} z=q(x) q(y) z-q(x) q(y, z) y-q(y) q(x, z) x,
$$

which continues to be symmetric in $x$ and $y$. We do not know whether a similar formula holds in general.

\section{Albert algebras}

In view of the results derived so far, particularly (7.4/2), one is tempted to conjecture that, given elements $x, y, z$ satisfying $x \circ y=0$ in a cubic Jordan algebra, there exists a formula, in terms of norm, trace and adjoint, for the expression $U_{x} U_{y} z$ that is symmetric in $x$ and $y$. Unfortunately, we have not been able to confirm this, even if the base ring is a field and low (positive) characteristics are excluded. Instead, we have to settle with the following theorem, giving an affirmative answer to Question (111) for arbitrary Albert algebras.

Theorem 8.1. Let $J$ be an Albert algebra over a field $F$. If $x, y \in J$ satisfy the relation $x \circ y=0$, then the operators $U_{x}$ and $U_{y}$ commute.

The proof of this theorem requires a few preparations that will be developed as we go along.

8.2. Initiating the proof of Theorem 8.1. Changing scalars to the algebraic closure of $F$, we may assume that $J$ is split. By the Jacobson embedding theorem [5. Theorem IX.11], which is valid in all characteristics [16, $x$ is contained in a (unital) subalgebra of $J$ isomorphic to $A^{+}, A:=\operatorname{Mat}_{3}(F)$. We may therefore realize $J$ as a first Tits construction via

$$
J=J(A, 1)=A \oplus A j_{1} \oplus A j_{2}
$$


as in Section 4.4 with $\mu=1$ such that

$$
x=x_{0}, \quad y=y_{0}+y_{1} j_{1}+y_{2} j_{2} \quad\left(x_{0}, y_{0}, y_{1}, y_{2} \in A\right) .
$$

We wish to study the implication

$$
x \circ y=0 \Longrightarrow \forall z \in J: U_{x} U_{y} z=U_{y} U_{x} z,
$$

and we will do so not only for $A=\operatorname{Mat}_{3}(F)$ as above. Instead, we will assume from now on that $A$ be any separable alternative algebra of degree 3 over $F$, with generic norm $N$, generic trace $T$ and adjoint $x \mapsto x^{\sharp}$. The reason for working in this more general context will become apparent in Corollary 8.12 below.

By linearity and (1), we may assume $z=z_{0} \in A$ or $z=z_{i} j_{i}, z_{i} \in A, i=1,2$, in (3). If $\tau: A \rightarrow A$ is an anti-automorphism (e.g., $z \mapsto z^{t}$ in the special case $A=\operatorname{Mat}_{3}(F)$ considered before), it is readily checked that the linear bijection

$$
J \stackrel{\sim}{\longrightarrow} J, \quad z_{0}+z_{1} j_{1}+z_{2} j_{2} \longmapsto \tau\left(z_{0}\right)+\tau\left(z_{2}\right) j_{1}+\tau\left(z_{1}\right) j_{2}
$$

preserves base points, norms and adjoints, and hence is an isomorphism. For the purpose of proving Theorem 8.1 it will therefore be enough to consider the cases $z=z_{0}$ and $z=z_{1} j_{1}, z_{i} \in A, i=0,1$ in (3).

Lemma 8.3. The following conditions are equivalent:

(i) $x \circ y=0$.

(ii) $x \circ y_{0}=x \circ\left(y_{1} j_{1}\right)=x \circ\left(y_{2} j_{2}\right)=0$.

(iii) $x_{0} \circ y_{0}=0, x_{0} y_{1}=T\left(x_{0}\right) y_{1}, y_{2} x_{0}=T\left(x_{0}\right) y_{2}$.

Proof. From (4.4/2), (8.22) we conclude $x \times y=x_{0} \times y_{0}-\left(x_{0} y_{1}\right) j_{1}-\left(y_{2} x_{0}\right) j_{2}$, and (4.28), 4.43), 4.440 imply

$$
\begin{aligned}
x \circ y= & x_{0} \times y_{0}-\left(x_{0} y_{1}\right) j_{1}-\left(y_{2} x_{0}\right) j_{2}+T\left(x_{0}\right) y_{0}+T\left(x_{0}\right)\left(y_{1} j_{1}\right) \\
& +T\left(x_{0}\right)\left(y_{2} j_{2}\right)+T\left(y_{0}\right) x_{0}-\left(T\left(x_{0}\right) T\left(y_{0}\right)-T\left(x_{0}, y_{0}\right)\right) 1 \\
= & x_{0} \circ y_{0}+\left(T\left(x_{0}\right) y_{1}-x_{0} y_{1}\right) j_{1}+\left(T\left(x_{0}\right) y_{2}-y_{2} x_{0}\right) j_{2} .
\end{aligned}
$$

Hence $x \circ y_{0}=x_{0} \circ y_{0} \in A, x \circ\left(y_{i} j_{i}\right) \in A j_{i}$ for $i=1,2$, and the assertion follows.

8.4. Iterated $U$-operators. Applying (4.25), (4.43), 4.411), (4.42), a straightforward computation shows, for all $z_{0}, z_{1}, z_{2} \in A$,

$$
\begin{aligned}
U_{x} z_{0}= & U_{x_{0}} z_{0}=x_{0} z_{0} x_{0}, \\
U_{x}\left(z_{1} j_{1}\right)= & \left(x_{0}^{\sharp} z_{1}\right) j_{1}, \\
U_{x}\left(z_{2} j_{2}\right)= & \left(z_{2} x_{0}^{\sharp}\right) j_{2}, \\
U_{y} z_{0}= & \left(U_{y_{0}} z_{0}+\left(y_{1} y_{2}\right) \times z_{0}\right)+\left(T\left(y_{0} z_{0}\right) y_{1}+z_{0} y_{2}^{\sharp}\right. \\
& \left.-z_{0}\left(y_{0} y_{1}\right)\right) j_{1}+\left(T\left(y_{0} z_{0}\right) y_{2}+y_{1}^{\sharp} z_{0}-\left(y_{2} y_{0}\right) z_{0}\right) j_{2}, \\
U_{y}\left(z_{1} j_{1}\right)= & \left(T\left(y_{2} z_{1}\right) y_{0}+z_{1} y_{1}^{\sharp}-z_{1}\left(y_{2} y_{0}\right)\right)+\left(T\left(y_{2} z_{1}\right) y_{1}+y_{0}^{\sharp} z_{1}\right. \\
& \left.-\left(y_{1} y_{2}\right) z_{1}\right) j_{1}+\left(U_{y_{2}} z_{1}+\left(y_{0} y_{1}\right) \times z_{1}\right) j_{2} .
\end{aligned}
$$


Making repeated use of (11)-(5), we now obtain

$$
\begin{aligned}
U_{x} U_{y} z_{0}= & \left(U_{x_{0}} U_{y_{0}} z_{0}+x_{0}\left(\left(y_{1} y_{2}\right) \times z_{0}\right) x_{0}\right) \\
& +\left(T\left(y_{0} z_{0}\right) x_{0}^{\sharp} y_{1}+x_{0}^{\sharp}\left(z_{0} y_{2}^{\sharp}\right)-x_{0}^{\sharp}\left(z_{0}\left(y_{0} y_{1}\right)\right)\right) j_{1} \\
& +\left(T\left(y_{0} z_{0}\right) y_{2} x_{0}^{\sharp}+\left(y_{1}^{\sharp} z_{0}\right) x_{0}^{\sharp}-\left(\left(y_{2} y_{0}\right) z_{0}\right) x_{0}^{\sharp}\right) j_{2}, \\
U_{y} U_{x} z_{0}= & \left(U_{y_{0}} U_{x_{0}} z_{0}+\left(y_{1} y_{2}\right) \times\left(x_{0} z_{0} x_{0}\right)\right) \\
& +\left(T\left(\left(x_{0} y_{0} x_{0}\right) z_{0}\right) y_{1}+\left(x_{0} z_{0} x_{0}\right) y_{2}^{\sharp}-\left(x_{0} z_{0} x_{0}\right)\left(y_{0} y_{1}\right)\right) j_{1} \\
& +\left(T\left(\left(x_{0} y_{0} x_{0}\right) z_{0}\right) y_{2}+y_{1}^{\sharp}\left(x_{0} z_{0} x_{0}\right)-\left(y_{2} y_{0}\right)\left(x_{0} z_{0} x_{0}\right)\right) j_{2}, \\
U_{x} U_{y}\left(z_{1} j_{1}\right)= & \left(T\left(y_{2} z_{1}\right) x_{0} y_{0} x_{0}+x_{0}\left(z_{1} y_{1}^{\sharp}\right) x_{0}-x_{0}\left(z_{1}\left(y_{2} y_{0}\right)\right) x_{0}\right) \\
& +\left(T\left(y_{2} z_{1}\right) x_{0}^{\sharp} y_{1}+x_{0}^{\sharp}\left(y_{0}^{\sharp} z_{1}\right)-x_{0}^{\sharp}\left(\left(y_{1} y_{2}\right) z_{1}\right)\right) j_{1} \\
& +\left(\left(y_{2} z_{1} y_{2}\right) x_{0}^{\sharp}+\left(\left(y_{0} y_{1}\right) \times z_{1}\right) x_{0}^{\sharp}\right) j_{2}, \\
U_{y} U_{x}\left(z_{1} j_{1}\right)= & \left(T\left(y_{2} x_{0}^{\sharp} z_{1}\right) y_{0}+\left(x_{0}^{\sharp} z_{1}\right) y_{1}^{\sharp}-\left(x_{0}^{\sharp} z_{1}\right)\left(y_{2} y_{0}\right)\right) \\
& +\left(T\left(y_{2} x_{0}^{\sharp} z_{1}\right) y_{1}+y_{0}^{\sharp}\left(x_{0}^{\sharp} z_{1}\right)-\left(y_{1} y_{2}\right)\left(x_{0}^{\sharp} z_{1}\right)\right) j_{1} \\
& +\left(y_{2}\left(x_{0}^{\sharp} z_{1}\right) y_{2}+\left(y_{0} y_{1}\right) \times\left(x_{0}^{\sharp} z_{1}\right)\right) j_{2} .
\end{aligned}
$$

Lemma 8.5. The following conditions are equivalent:

(i) Whenever $x \in A \subseteq J$ and $y \in J$ satisfy $x \circ y=0$, then the linear operators $U_{x} U_{y}$ and $U_{y} U_{x}$ agree on $A \oplus A j_{1}$.

(ii) Whenever $x_{0}, y_{0}, y_{1}, y_{2} \in A$ satisfy the relations

$$
x_{0} \circ y_{0}=0, \quad x_{0} y_{1}=T\left(x_{0}\right) y_{1}, \quad y_{2} x_{0}=T\left(x_{0}\right) y_{2},
$$

then

$$
\begin{aligned}
x_{0}\left(\left(y_{1} y_{2}\right) \times z\right) x_{0} & =\left(y_{1} y_{2}\right) \times\left(x_{0} z x_{0}\right), \\
T\left(y_{0} z\right) x_{0}^{\sharp} y_{1}-x_{0}^{\sharp}\left(z\left(y_{0} y_{1}\right)\right) & =T\left(\left(x_{0} y_{0} x_{0}\right) z\right) y_{1}-\left(x_{0} z x_{0}\right)\left(y_{0} y_{1}\right), \\
x_{0}^{\sharp}\left(z y_{2}^{\sharp}\right) & =\left(x_{0} z x_{0}\right) y_{2}^{\sharp}, \\
T\left(y_{0} z\right) y_{2} x_{0}^{\sharp}-\left(\left(y_{2} y_{0}\right) z\right) x_{0}^{\sharp} & =T\left(\left(x_{0} y_{0} x_{0}\right) z\right) y_{2}-\left(y_{2} y_{0}\right)\left(x_{0} z x_{0}\right), \\
\left(y_{1}^{\sharp} z\right) x_{0}^{\sharp} & =y_{1}^{\sharp}\left(x_{0} z x_{0}\right), \\
T\left(y_{2} z\right) x_{0} y_{0} x_{0}-x_{0}\left(z\left(y_{2} y_{0}\right)\right) x_{0} & =T\left(y_{2} x_{0}^{\sharp} z\right) y_{0}-\left(x_{0}^{\sharp} z\right)\left(y_{2} y_{0}\right), \\
x_{0}\left(z y_{1}^{\sharp}\right) x_{0} & =\left(x_{0}^{\sharp} z\right) y_{1}^{\sharp}, \\
T\left(y_{2} z\right) x_{0}^{\sharp} y_{1}-x_{0}^{\sharp}\left(\left(y_{1} y_{2}\right) z\right) & =T\left(y_{2} x_{0}^{\sharp} z\right) y_{1}-\left(y_{1} y_{2}\right)\left(x_{0}^{\sharp} z\right), \\
x_{0}^{\sharp}\left(y_{0}^{\sharp} z\right) & =y_{0}^{\sharp}\left(x_{0}^{\sharp} z\right), \\
\left(y_{2} z y_{2}\right) x_{0}^{\sharp} & =y_{2}\left(x_{0}^{\sharp} z\right) y_{2}, \\
\left(\left(y_{0} y_{1}\right) \times z\right) x_{0}^{\sharp} & =\left(y_{0} y_{1}\right) \times\left(x_{0}^{\sharp} z\right)
\end{aligned}
$$

for all $z \in A$. 
Proof. By Lemma 8.3, elements $x_{0}, y_{0}, y_{1}, y_{2} \in A$ satisfy (11) if and only if $x=x_{0} \in$ $A \subseteq J$ and $y=y_{0}+y_{1} j_{1}+y_{2} j_{2} \in J$ satisfy $x \circ y=0$. In this case, since $A^{+}$is special, Section 4.1 implies $U_{x_{0}} U_{y_{0}}=U_{y_{0}} U_{x_{0}}$ on $A$. Hence the assertion follows from inspecting equations (8.4[6) - 8.4[9).

8.6. Continuing the proof of Theorem 8.1. Until the end of the proof we assume that $A$ is associative. Combining the reductions carried out in Section 8.2 with Lemma 8.5, the implication (8.23) will follow once we have shown that 8.5]1] implies 8.512 - 8.512), so let us suppose from now on that 8.511 holds. By passing to $A^{\text {op }}$ if necessary, (8.5I5) (resp. (8.5[4)) follows from (8.5]3) (resp. (8.5]6)). We are thus reduced to showing

$$
\text { (8.512), (8.513), (8.566)-(8.512). }
$$

Proof of (8.5/2). Applying (4.211), (8.5/1) and (4.3/4) first for $v_{2}=1$ and then for $u_{2}=1$, we obtain

$$
\begin{aligned}
x_{0}\left(\left(y_{1} y_{2}\right) \times z\right) x_{0}= & T\left(x_{0}\right)\left(\left(y_{1} y_{2}\right) \times z\right) x_{0}-\left(x_{0} \times 1\right)\left(\left(y_{1} y_{2}\right) \times z\right) x_{0} \\
= & T\left(x_{0}\right)\left(\left(y_{1} y_{2}\right) \times z\right) x_{0}-\left(\left(y_{1} y_{2} x_{0}\right) \times z\right) x_{0}-\left(\left(y_{1} y_{2}\right) \times\left(z x_{0}\right)\right) x_{0} \\
= & -\left(\left(y_{1} y_{2}\right) \times\left(z x_{0}\right)\right) x_{0} \\
= & -T\left(x_{0}\right)\left(\left(y_{1} y_{2}\right) \times\left(z x_{0}\right)\right)+\left(\left(y_{1} y_{2}\right) \times\left(z x_{0}\right)\right)\left(x_{0} \times 1\right) \\
= & -T\left(x_{0}\right)\left(\left(y_{1} y_{2}\right) \times\left(z x_{0}\right)\right)+\left(x_{0} y_{1} y_{2}\right) \times\left(z x_{0}\right) \\
& +\left(x_{0} z x_{0}\right) \times\left(y_{1} y_{2}\right) \\
= & \left(y_{1} y_{2}\right) \times\left(x_{0} z x_{0}\right) .
\end{aligned}
$$

Proof of (8.5).31). This time we combine (4.211) with (4.322) and conclude

$$
\begin{aligned}
T\left(y_{0} z\right) x_{0}^{\sharp} y_{1}-x_{0}^{\sharp} z y_{0} y_{1} & =x_{0}^{\sharp}\left(T\left(z y_{0}\right) 1-z y_{0}\right) y_{1}=x_{0}^{\sharp}\left(\left(z y_{0}\right) \times 1\right) y_{1} \\
& =\left(\left(z y_{0} x_{0}\right) \times x_{0}\right) y_{1},
\end{aligned}
$$

so by (4.28) and (8.511) we have

$$
\begin{aligned}
T\left(y_{0} z\right) x_{0}^{\sharp} y_{1}-x_{0}^{\sharp} z y_{0} y_{1}= & z y_{0} x_{0}^{2} y_{1}+x_{0} z y_{0} x_{0} y_{1}-T\left(z y_{0} x_{0}\right) x_{0} y_{1} \\
& -T\left(x_{0}\right) z y_{0} x_{0} y_{1}+T\left(z y_{0} x_{0}\right) T\left(x_{0}\right) y_{1}-T\left(z y_{0} x_{0}^{2}\right) y_{1} \\
= & T\left(x_{0}\right)^{2} z y_{0} y_{1}-x_{0} z x_{0} y_{0} y_{1}-T\left(x_{0}\right) T\left(z y_{0} x_{0}\right) y_{1} \\
& -T\left(x_{0}\right)^{2} z y_{0} y_{1}+T\left(z y_{0} x_{0}\right) T\left(x_{0}\right) y_{1}+T\left(x_{0} y_{0} x_{0} z\right) y_{1} \\
= & T\left(x_{0} y_{0} x_{0} z\right) y_{1}-x_{0} z x_{0} y_{0} y_{1} .
\end{aligned}
$$

Proof of (8.5) 10). Using (4.311) and (8.511) we obtain $x_{0}^{\sharp} y_{0}^{\sharp} z=\left(y_{0} x_{0}\right)^{\sharp} z=\left(-x_{0} y_{0}\right)^{\sharp} z$ $=\left(x_{0} y_{0}\right)^{\sharp} z=y_{0}^{\sharp} x_{0}^{\sharp} z$, hence (8.5]10).

In view of (1), it remains to verify

$$
\text { (8.5[6) - (8.519), (8.5[11), (8.5[12). }
$$

In order to do so, we require a few further preparations.

Lemma 8.7. Suppose $u, v \in A$ satisfy $u v=0$. Then

$$
u^{\sharp} v=T\left(u^{\sharp}\right) v, \quad u v^{\sharp}=T\left(v^{\sharp}\right) u .
$$

Moreover, $u^{\sharp}=0$ or $v^{\sharp}=0$. 
Proof. By (4.27) $u^{\sharp} v=u^{2} v-T(u) u v+T\left(u^{\sharp}\right) 1 v=T\left(u^{\sharp}\right) v$, giving the first equation of (11). The second follows from the first by passing to $A^{\text {op }}$. Now suppose $v^{\sharp} \neq 0$. Then $u$ cannot be invertible, forcing $N(u)=0$. Taking adjoints in the first equation of (11) and applying (4.22), (4.311), we therefore obtain $0=N(u) v^{\sharp} u=v^{\sharp} u^{\sharp \sharp}=$ $\left(u^{\sharp} v\right)^{\sharp}=T\left(u^{\sharp}\right)^{2} v^{\sharp}$, and hence $T\left(u^{\sharp}\right)=0$. For any $a \in A$ we have auv $=0$, so the preceding considerations apply to $a u$ in place of $u$ and yield $T\left(u^{\sharp} a^{\sharp}\right)=0$. Linearizing and applying (4.21), we conclude $0=T\left(u^{\sharp}(1 \times a)\right)=T\left(u^{\sharp}\right) T(a)-$ $T\left(u^{\sharp} a\right)=-T\left(u^{\sharp} a\right)$, and hence $u^{\sharp}=0$ since $A$ was assumed to be separable (cf. Sections 4.3 and 8.2 .

Lemma 8.8. With the notation and assumptions of Section 8.6 we have

$$
\begin{aligned}
x_{0}^{\sharp} y_{1}=T\left(x_{0}^{\sharp}\right) y_{1}, & y_{2} x_{0}^{\sharp}=T\left(x_{0}^{\sharp}\right) y_{2}, \\
y_{1}^{\sharp} x_{0}=-T\left(x_{0}\right) y_{1}^{\sharp}, & x_{0} y_{2}^{\sharp}=-T\left(x_{0}\right) y_{2}^{\sharp} .
\end{aligned}
$$

If $y_{1}^{\sharp} \neq 0$ or $y_{2}^{\sharp} \neq 0$, then

$$
x_{0}^{\sharp}=-T\left(x_{0}\right) x_{0} .
$$

Proof. By passing to $A^{\text {op }}$ if necessary, it suffices to establish the first equations of (11), (2) and to derive (3) under the assumption $y_{1}^{\sharp} \neq 0$.

Combining (4.27) with (8.51), we obtain $x_{0}^{\sharp} y_{1}=x_{0}^{2} y_{1}-T\left(x_{0}\right) x_{0} y_{1}+T\left(x_{0}^{\sharp}\right) 1 y_{1}=$ $T\left(x_{0}\right)^{2} y_{1}-T\left(x_{0}\right)^{2} y_{1}+T\left(x_{0}^{\sharp}\right) y_{1}$, hence the first equation of (11). Moreover, combining (4.21) with (4.32) yields

$$
\begin{aligned}
y_{1}^{\sharp} x_{0} & =T\left(x_{0}\right) y_{1}^{\sharp}-y_{1}^{\sharp}\left(x_{0} \times 1\right)=T\left(x_{0}\right) y_{1}^{\sharp}-\left(x_{0} y_{1}\right) \times y_{1} \\
& =T\left(x_{0}\right) y_{1}^{\sharp}-T\left(x_{0}\right) y_{1} \times y_{1}=T\left(x_{0}\right) y_{1}^{\sharp}-2 T\left(x_{0}\right) y_{1}^{\sharp},
\end{aligned}
$$

and this is the first equation of (2). It remains to prove (3) under the assumption $y_{1}^{\sharp} \neq 0$. Setting $\bar{x}_{0}:=T\left(x_{0}\right) 1-x_{0}$, we deduce $\bar{x}_{0} y_{1}=0$ from (8.51), and Lemma 8.7 implies $0=\bar{x}_{0}^{\sharp}=\left(T\left(x_{0}\right) 1-x_{0}\right)^{\sharp}=T\left(x_{0}\right)^{2} 1-T\left(x_{0}\right)\left(1 \times x_{0}\right)+x_{0}^{\sharp}=T\left(x_{0}\right)^{2} 1-$ $T\left(x_{0}\right)^{2} 1+T\left(x_{0}\right) x_{0}+x_{0}^{\sharp}$, and (3) follows.

Remark. The referee has noted that (3) does not follow if $y_{1}^{\sharp}=y_{2}^{\sharp}=0$ : with the usual matrix units $e_{i j} \in A:=\operatorname{Mat}_{3}(F)$, put $x_{0}=y_{1}=y_{2}=e_{11}, y_{0}=e_{22}$.

8.9. Returning to the proof of Theorem 8.1. We now proceed to establish the equations of (8.612).

Proof of (8.5,6). The equation is obvious for $y_{1}^{\sharp}=0$, allowing us to assume $y_{1}^{\sharp} \neq 0$. Then (8.813) holds, and if $T\left(x_{0}\right) \neq 0$, we conclude $y_{1}^{\sharp} x_{0} z x_{0}=-T\left(x_{0}\right)^{-1} y_{1}^{\sharp} x_{0}^{\sharp} z x_{0}=$ $-T\left(x_{0}\right)^{-1}\left(x_{0} y_{1}\right)^{\sharp} z x_{0}=-T\left(x_{0}\right)^{-1} T\left(x_{0}\right)^{2} y_{1}^{\sharp} z x_{0}=y_{1}^{\sharp} z x_{0}^{\sharp}$. Thus we are reduced to the case $T\left(x_{0}\right)=0$, which implies $x_{0}^{\sharp}=0$ by (8.83), hence $y_{1}^{\sharp} z x_{0}^{\sharp}=0$, and (4.25), (8.82) yield $y_{1}^{\sharp} x_{0} z x_{0}=y_{1}^{\sharp}\left(U_{x_{0}} z\right)=y_{1}^{\sharp} T\left(x_{0} z\right) x_{0}=-T\left(x_{0}\right) T\left(x_{0} z\right) y_{1}^{\sharp}=0$. 
Proof of (8.5). Consulting (4.21), 4.32), (8.511) and (4.28), we obtain

$$
\begin{aligned}
x_{0}^{\sharp} z y_{2}= & T\left(z y_{2}\right) x_{0}^{\sharp}-x_{0}^{\sharp}\left(\left(z y_{2}\right) \times 1\right)=T\left(z y_{2}\right) x_{0}^{\sharp}-\left(z y_{2} x_{0}\right) \times x_{0} \\
= & T\left(z y_{2}\right) x_{0}^{\sharp}-T\left(x_{0}\right)\left(z y_{2}\right) \times x_{0} \\
= & T\left(z y_{2}\right) x_{0}^{\sharp}-T\left(x_{0}\right) z y_{2} x_{0}-T\left(x_{0}\right) x_{0} z y_{2}+T\left(x_{0}\right) T\left(z y_{2}\right) x_{0} \\
& +T\left(x_{0}\right)^{2} z y_{2}-T\left(x_{0}\right)^{2} T\left(z y_{2}\right) 1+T\left(x_{0}\right) T\left(z y_{2} x_{0}\right) 1 \\
= & T\left(z y_{2}\right) x_{0}^{\sharp}-T\left(x_{0}\right)^{2} z y_{2}-T\left(x_{0}\right) x_{0} z y_{2}+T\left(x_{0}\right) T\left(z y_{2}\right) x_{0} \\
& +T\left(x_{0}\right)^{2} z y_{2}-T\left(x_{0}\right)^{2} T\left(z y_{2}\right) 1+T\left(x_{0}\right)^{2} T\left(z y_{2}\right) 1 \\
= & T\left(z y_{2}\right)\left(x_{0}^{\sharp}+T\left(x_{0}\right) x_{0}\right)-T\left(x_{0}\right) x_{0} z y_{2} .
\end{aligned}
$$

Combining this with (4.27) we deduce

$$
x_{0}^{\sharp} z y_{2}=T\left(z y_{2}\right) x_{0}^{2}+T\left(x_{0}^{\sharp}\right) T\left(z y_{2}\right) 1-T\left(x_{0}\right) x_{0} z y_{2},
$$

and (8.81) yields

$$
\begin{aligned}
T\left(y_{2} x_{0}^{\sharp} z\right) y_{0}-x_{0}^{\sharp} z y_{2} y_{0}= & T\left(x_{0}^{\sharp}\right) T\left(y_{2} z\right) y_{0}-T\left(z y_{2}\right) x_{0}^{2} y_{0} \\
& -T\left(x_{0}^{\sharp}\right) T\left(z y_{2}\right) y_{0}+T\left(x_{0}\right) x_{0} z y_{2} y_{0} \\
= & T\left(z y_{2}\right) x_{0} y_{0} x_{0}+x_{0} z y_{2} x_{0} y_{0} \\
= & T\left(y_{2} z\right) x_{0} y_{0} x_{0}-x_{0} z y_{2} y_{0} x_{0},
\end{aligned}
$$

as claimed.

Proof of (8.5). Again we may assume $y_{1}^{\sharp} \neq 0$, so (8.83) holds. Hence, by (8.812), $x_{0} z y_{1}^{\sharp} x_{0}=-T\left(x_{0}\right) x_{0} z y_{1}^{\sharp}=x_{0}^{\sharp} z y_{1}^{\sharp}$.

Proof of (8.5.9), 8.5,11). We begin by applying (8.811) and obtain

$$
T\left(y_{2} z\right) x_{0}^{\sharp} y_{1}-x_{0}^{\sharp} y_{1} y_{2} z=T\left(x_{0}^{\sharp}\right) T\left(y_{2} z\right) y_{1}-T\left(x_{0}^{\sharp}\right) y_{1} y_{2} z=T\left(y_{2} x_{0}^{\sharp} z\right) y_{1}-y_{1} y_{2} x_{0}^{\sharp} z,
$$

giving (8.5[9). Similarly, $y_{2} z y_{2} x_{0}^{\sharp}=T\left(x_{0}^{\sharp}\right) y_{2} z y_{2}=y_{2} x_{0}^{\sharp} z y_{2}$, giving (8.5[11).

We have thus verified all equations of (8.62) with the exception of (8.512), which turns out to be the most difficult. We begin with yet another technical result.

Lemma 8.10. Let $c \in A$ be an element satisfying $T(c)=1$ and $c^{\sharp}=0$.

(a) $c$ is an idempotent of $A$ with the Peirce decomposition

$$
A=A_{11} \oplus A_{12} \oplus A_{21} \oplus A_{22}, \quad A_{11}=F c,
$$

where $A_{i j}=c_{i} A c_{j}$ for $i, j=1,2, c_{1}:=c, c_{2}:=1-c$.

(b) $A_{22}^{+}$is the Jordan algebra of a pointed quadratic form. More precisely,

$$
A_{22}^{+}=J\left(A_{22}, z_{22} \mapsto T\left(z_{22}^{\sharp}\right), d\right), \quad d:=1-c,
$$

and the trace of $A$ restricts to the trace of $A_{22}^{+}$. Furthermore

$$
z_{22} \longmapsto \bar{z}_{22}=T\left(z_{22}\right) d-z_{22}=c \times z_{22}
$$

is the conjugation of $A_{22}^{+}$and an algebra involution of the associative algebra $A_{22}$. 
(c) The relations

$$
\begin{aligned}
c \times z_{12}=c \times z_{21} & =z_{12}^{\sharp}=z_{21}^{\sharp}=0, \\
z_{22}^{\sharp} & =T\left(z_{22}^{\sharp}\right) c, \\
T\left(z_{12}\right) & =T\left(z_{21}\right)=0, \\
z_{12} \times z_{22} & =-z_{12} \bar{z}_{22}, \\
z_{21} \times z_{22} & =-\bar{z}_{22} z_{21}, \\
z_{12} \times z_{21} & =-\bar{z}_{21} z_{12}
\end{aligned}
$$

hold for all $z_{i j} \in A_{i j}, i, j=1,2,(i, j) \neq(1,1)$.

Proof. (a) By (4.277), $c \in A$ is an idempotent, and if $x \in A$ satisfies $c x=x=x c$, then (4.25) yields $x=c x c=U_{c} x=T(c x) c$, proving (1).

(b) The first part is basically just Faulkner's lemma [3, Lemma 1.5]. Since $A_{22}$ is an associative $F$-algebra of degree 2 in the sense of McCrimmon 13, its conjugation is an algebra involution by (4.24) and [13, Theorem 1.1].

(c) These relations follow from a number of easy computations, using (2), (4.277), (4.28), (4.33), (4.32), (4.311): $c \times z_{22}=c z_{22}+z_{22} c-T(c) z_{22}-T\left(z_{22}\right) c+$ $\left(T(c) T\left(z_{22}\right)-T\left(c z_{22}\right)\right) 1=-z_{22}-T\left(z_{22}\right) c+T\left(z_{22}\right) 1=T\left(z_{22}\right) d-z_{22}=\bar{z}_{22}$, giving (3) ; $c \times z_{12}=c \times c z_{12}=\left(1 \times z_{12}\right) c^{\sharp}=0, c \times z_{21}=c \times\left(z_{21} c\right)=c^{\sharp}\left(1 \times z_{21}\right)$ $=0, z_{12}^{\sharp}=\left(c z_{12}\right)^{\sharp}=z_{12}^{\sharp} c^{\sharp}=0, z_{21}^{\sharp}=\left(z_{21} c\right)^{\sharp}=c^{\sharp} z_{21}^{\sharp}=0$, giving (41); $z_{22}^{\sharp}=$ $z_{22}^{2}-T\left(z_{22}\right) z_{22}+T\left(z_{22}^{\sharp}\right) 1=-T\left(z_{22}^{\sharp}\right) d+T\left(z_{22}^{\sharp}\right) 1=T\left(z_{22}^{\sharp}\right) c$, giving (5); $T\left(z_{12}\right)=$ $T\left(c z_{12}\right)=T\left(z_{12} c\right)=0, T\left(z_{21}\right)=T\left(z_{21} c\right)=T\left(c z_{21}\right)=0$, giving (6) $; z_{12} \times z_{22}=$ $z_{12} z_{22}+z_{22} z_{12}-T\left(z_{12}\right) z_{22}-T\left(z_{22}\right) z_{12}+\left(T\left(z_{12}\right) T\left(z_{22}\right)-T\left(z_{12} z_{22}\right)\right) 1=z_{12} z_{22}-$ $T\left(z_{22}\right) z_{12} d=-z_{12} \bar{z}_{22}$, giving (7); $z_{21} \times z_{22}=z_{21} z_{22}+z_{22} z_{21}-T\left(z_{21}\right) z_{22}-T\left(z_{22}\right) z_{21}+$ $\left(T\left(z_{21}\right) T\left(z_{22}\right)-T\left(z_{21} z_{22}\right)\right) 1=z_{22} z_{21}-T\left(z_{22}\right) d z_{21}=-\bar{z}_{22} z_{21}$, giving (8); ; and finally, by (11),

$$
\begin{aligned}
z_{12} \times z_{21}= & z_{12} z_{21}+z_{21} z_{12}-T\left(z_{12}\right) z_{21}-T\left(z_{21}\right) z_{12} \\
& +\left(T\left(z_{12}\right) T\left(z_{21}\right)-T\left(z_{12} z_{21}\right)\right) 1 \\
= & T\left(z_{12} z_{21}\right) c+z_{21} z_{12}-T\left(z_{12} z_{21}\right) 1 \\
= & z_{21} z_{12}-T\left(z_{21} z_{12}\right) d=-\overline{z_{21} z_{12}}
\end{aligned}
$$

giving (9).

8.11. Finishing the proof of Theorem 8.1. We are now prepared to prove 8.5[12). To this end, we proceed in several steps.

$\mathbf{1}^{0}$. We reduce to the case $y_{1}^{\sharp}=0$. Indeed, if $y_{1}^{\sharp} \neq 0$, then (8.8I3) holds and (4.3) yields

$$
\begin{aligned}
\left(\left(y_{0} y_{1}\right) \times z\right) x_{0}^{\sharp} & =\left(x_{0} y_{0} y_{1}\right) \times\left(x_{0} z\right)=-\left(y_{0} x_{0} y_{1}\right) \times\left(x_{0} z\right) \\
& =-\left(y_{0} y_{1}\right) \times\left(T\left(x_{0}\right) x_{0} z\right)=\left(y_{0} y_{1}\right) \times\left(x_{0}^{\sharp} z\right),
\end{aligned}
$$

as claimed.

$\mathbf{2}^{0}$. Being allowed to assume $y_{1}^{\sharp}=0$ by $1^{0}$, we next reduce to the case $T\left(y_{1}\right)=1$, forcing $y_{1} \in A$ to be an idempotent as in Lemma 8.10. To see this, we assume 
(8.511) implies (8.5]12) if $y_{1}^{\sharp}=0$ and $T\left(y_{1}\right)=1$. Then suppose $y_{1}$ with (8.5]1) and $y_{1}^{\sharp}=0$ is arbitrary, consider the subspace

$$
I:=\left\{v \in A \mid(v \times z) x_{0}^{\sharp}=v \times\left(x_{0}^{\sharp} z\right) \text { for all } z \in A\right\}
$$

of $A$ and let $v \in I, w \in A$. Then (4.34) gives, for all $z \in A$,

$$
\begin{aligned}
((v w) \times z) x_{0}^{\sharp}+(v \times(z w)) x_{0}^{\sharp} & =(w \times 1)(v \times z) x_{0}^{\sharp}=(w \times 1)\left(\left(v \times\left(x_{0}^{\sharp} z\right)\right)\right. \\
& =(v w) \times\left(x_{0}^{\sharp} z\right)+\left(v \times\left(x_{0}^{\sharp} z w\right)\right) \\
& =(v w) \times\left(x_{0}^{\sharp} z\right)+(v \times(z w)) x_{0}^{\sharp} ;
\end{aligned}
$$

hence $v w \in I$, so $I \subseteq A$ is a right ideal. Now, by non-degeneracy of $T$, some $w \in A$ has $T(c)=1$ with $c:=y_{1} w$. Since, in addition, $c^{\sharp}=w^{\sharp} y_{1}^{\sharp}=0$,

$$
c y_{1}=y_{1} w y_{1}=T(c) y_{1}-y_{1}^{\sharp} \times w=y_{1}, \quad x_{0} c=x_{0} y_{1} w=T\left(x_{0}\right) y_{1} w=T\left(x_{0}\right) c,
$$

we conclude $y_{0} c \in I$ (by the special case whose validity we have assumed), forcing $y_{0} y_{1}=y_{0} c y_{1} \in I$ since $I \subseteq A$ is a right ideal. Hence (8.512) holds.

$\mathbf{3}^{0}$. For the rest of the proof, we may and always will assume that the element $y_{1}=c \in A$ satisfies $T(c)=1, c^{\sharp}=0$, allowing us to adopt the notation of Lemma 8.10. Since $x_{0} c=T\left(x_{0}\right) c$ by (8.511), we have, by (8.1011), 8.106),

$$
x_{0}=T\left(x_{0}\right) c+x_{12}+x_{22}, \quad x_{12} \in A_{12}, \quad x_{22} \in A_{22}, \quad T\left(x_{22}\right)=0 .
$$

This and (8.10/2), 8.10]3) yield

$$
x_{22}^{2}=-T\left(x_{22}^{\sharp}\right) d, \quad \bar{x}_{22}=-x_{22} .
$$

Write

(3) $y_{0}=\beta c+y_{12}+y_{21}+y_{22}, \quad \beta \in F, \quad y_{i j} \in A_{i j} \quad(i, j=1,2,(i, j) \neq(1,1))$.

Comparing the Peirce components of $x_{0} y_{0}=-y_{0} x_{0}$ (cf. (8.5]1) ), we conclude

$$
\begin{aligned}
T\left(x_{12} y_{21}\right) & =-2 \beta T\left(x_{0}\right), \\
T\left(x_{0}\right) y_{12}+y_{12} x_{22} & =-\beta x_{12}-x_{12} y_{22}, \\
x_{22} y_{21} & =-T\left(x_{0}\right) y_{21}, \\
x_{22} y_{22}+y_{22} x_{22} & =-y_{21} x_{12} .
\end{aligned}
$$

Next we observe

$$
y_{0} y_{1}=y_{0} c=\beta c+y_{21} \in A_{11}+A_{21}
$$

by (3). Finally, we compute the Peirce components of $x_{0}^{\sharp}$ with the aid of (1), (2), (8.104), (8.1015), 88.1013), (8.1017) to obtain

$$
x_{0}^{\sharp}=T\left(x_{22}^{\sharp}\right) c+x_{12} x_{22}-T\left(x_{0}\right) x_{22} .
$$

In order to verify (8.512), we are reduced by linearity in $z$ to the following cases, the first one adopting a simplification due to the referee. $4^{0} . z \in A_{11}+A_{21}$. Since

$$
\left(A_{11}+A_{21}\right)^{\sharp}=A_{11}^{\sharp}+A_{11} \times A_{21}+A_{21}^{\sharp}=\{0\}
$$


by (8.104), we deduce $\left(y_{0} y_{1}\right) \times z=0$ from (8). On the other hand, by (9),

$$
x_{0}^{\sharp} z \in\left(T\left(x_{22}^{\sharp}\right) c+x_{12} x_{22}-T\left(x_{0}\right) x_{22}\right)\left(A_{11}+A_{21}\right) \subseteq A_{11}+A_{21},
$$

so (10) and again (8.1044) yield $\left(y_{0} y_{1}\right) \times\left(x_{0}^{\sharp} z\right)=0$ as well, and the proof of 8.5]12) is complete in this case.

$5^{0} . z=z_{12} \in A_{12}$. Then by (8) and (8.104), (8.109), $\left(y_{0} y_{1}\right) \times z=\left(\beta c+y_{21}\right) \times z_{12}=$ $-\overline{y_{21} z_{12}}$, forcing

(11) $\left(\left(y_{0} y_{1}\right) \times z\right) x_{0}^{\sharp}=-\overline{y_{21} z_{12}}\left(T\left(x_{22}^{\sharp}\right) c+x_{12} x_{22}-T\left(x_{0}\right) x_{22}\right)=T\left(x_{0}\right) \overline{y_{21} z_{12}} x_{22}$.

On the other hand, $x_{0}^{\sharp} z=\left(T\left(x_{22}^{\sharp}\right) c+x_{12} x_{22}-T\left(x_{0}\right) x_{22}\right) z_{12}=T\left(x_{22}^{\sharp}\right) z_{12}$, which implies

$$
\left(y_{0} y_{1}\right) \times\left(x_{0}^{\sharp} z\right)=T\left(x_{22}^{\sharp}\right)\left(\beta c+y_{21}\right) \times z_{12}=-T\left(x_{22}^{\sharp}\right) \overline{y_{21} z_{12}} .
$$

Taking conjugates in (11), (12) and invoking (2), we therefore have to show

$$
T\left(x_{0}\right) x_{22} y_{21}=T\left(x_{22}^{\sharp}\right) y_{21} .
$$

But from (2), (6) we conclude that $T\left(x_{0}\right) x_{22} y_{21}=-x_{22}^{2} y_{21}=T\left(x_{22}^{\sharp}\right) d y_{21}=T\left(x_{22}^{\sharp}\right) y_{21}$, and (13) holds.

$6^{0} . z=z_{22} \in A_{22}$. Then by (8) and (8.1013), (8.1018),

$$
\left(y_{0} y_{1}\right) \times z=\left(\beta c+y_{21}\right) \times z_{22}=-\bar{z}_{22} y_{21}+\beta \bar{z}_{22},
$$

which implies, using (9),

$$
\left(\left(y_{0} y_{1}\right) \times z\right) x_{0}^{\sharp}=\left(-\bar{z}_{22} y_{21}+\beta \bar{z}_{22}\right)\left(T\left(x_{22}^{\sharp}\right) c+x_{12} x_{22}-T\left(x_{0}\right) x_{22}\right),
$$

and hence

$$
\left(\left(y_{0} y_{1}\right) \times z\right) x_{0}^{\sharp}=-T\left(x_{22}^{\sharp}\right) \bar{z}_{22} y_{21}-\bar{z}_{22} y_{21} x_{12} x_{22}-\beta T\left(x_{0}\right) \bar{z}_{22} x_{22} .
$$

On the other hand, again by (9),

$$
x_{0}^{\sharp} z=\left(T\left(x_{22}^{\sharp}\right) c+x_{12} x_{22}-T\left(x_{0}\right) x_{22}\right) z_{22}=x_{12} x_{22} z_{22}-T\left(x_{0}\right) x_{22} z_{22},
$$

which by Lemma 8.10, particularly (8.103), (8.1018), (8.109) and (2), implies

$$
\begin{aligned}
\left(y_{0} y_{1}\right) \times\left(x_{0}^{\sharp} z\right) & =\left(\beta c+y_{21}\right) \times\left(x_{12} x_{22} z_{22}-T\left(x_{0}\right) x_{22} z_{22}\right) \\
& =-\beta T\left(x_{0}\right) \overline{x_{22} z_{22}}-\overline{y_{21} x_{12} x_{22} z_{22}}+T\left(x_{0}\right) \overline{x_{22} z_{22}} y_{21} \\
& =-T\left(x_{0}\right) \bar{z}_{22} x_{22} y_{21}+\beta T\left(x_{0}\right) \bar{z}_{22} x_{22}+\bar{z}_{22} x_{22} \overline{y_{21} x_{12}} .
\end{aligned}
$$

Comparing this with (14), we see that it suffices to show

$$
T\left(x_{22}^{\sharp}\right) y_{21}=T\left(x_{0}\right) x_{22} y_{21}, \quad y_{21} x_{12} x_{22}+2 \beta T\left(x_{0}\right) x_{22}+x_{22} \overline{y_{21} x_{12}}=0 .
$$


Here the first equation agrees with (13), while the second one follows from (7), (4), (2) and

$$
\begin{aligned}
y_{21} x_{12} x_{22} & +2 \beta T\left(x_{0}\right) x_{22}+x_{22} \overline{y_{21} x_{12}} \\
& =-x_{22} y_{22} x_{22}-y_{22} x_{22}^{2}-T\left(y_{21} x_{12}\right) x_{22}+x_{22} \overline{y_{21} x_{12}} \\
& =-x_{22} y_{22} x_{22}+T\left(x_{22}^{\sharp}\right) y_{22}-x_{22}\left(T\left(y_{21} x_{12}\right) d-\overline{y_{21} x_{12}}\right) \\
& =-x_{22} y_{22} x_{22}+T\left(x_{22}^{\sharp}\right) y_{22}-x_{22} y_{21} x_{12} \\
& =-x_{22} y_{22} x_{22}+T\left(x_{22}^{\sharp}\right) y_{22}+x_{22}^{2} y_{22}+x_{22} y_{22} x_{22} \\
& =T\left(x_{22}^{\sharp}\right) y_{22}-T\left(x_{22}^{\sharp}\right) y_{22}=0 .
\end{aligned}
$$

This concludes the proof of $(8.5[12)$ and hence of Theorem 8.1 ,

Corollary 8.12. Let $A$ be a separable alternative algebra of degree 3 over $F$, with generic norm $N$, generic trace $T$, and adjoint $x \mapsto x^{\sharp}$. If $x_{0}, y_{0}, y_{1}, y_{2} \in A$ satisfy (8.51), then the equations (8.5) 2) -(8.512) hold.

Proof. By hypothesis, the cubic Jordan algebra $J(A, 1)$ over $F$ is either special or Albert. In any event, we may conclude from Section 4.1 and Theorem 8.1 that Lemma 8.5 (i) holds. Hence so does Lemma 8.5 (ii).

Remark. It could be argued with some justification that, since equations (8.5/2)(8.512) are of a purely technical nature, Corollary 8.12 is of little intrinsic value. Yet in view of the fact that our proof of these equations (under the hypothesis (8.5]1)) relies heavily on $A$ being an associative algebra, the extension in Corollary 8.12 to the alternative case by appealing to the first Tits construction of Albert algebras could be of some interest from a methodological point of view. The fact that we were able to carry out this extension with virtually no extra cost may serve as a justification to include it here. Incidentally, the only alternative algebras to which Corollary 8.12 applies but which are not associative have the form $A=F \oplus C$, with $C$ an octonion algebra over $F$.

\section{Non-Unital Jordan ALGEBRAS}

After the preceding preparations, we are finally ready to tackle the main result of the paper. We begin by recalling the basic definitions.

9.1. The concept of a non-unital Jordan algebra. Following McCrimmon [12, we define a non-unital (quadratic) Jordan algebra over $k$ as a $k$-module $J$ together with two quadratic maps $J \rightarrow J, x \mapsto x^{2}$ (the squaring) and $U: J \rightarrow \operatorname{End}_{k}(J)$, $x \mapsto U_{x}$ (the $U$-operator) such that, setting

$$
x \circ y:=V_{x} y:=(x+y)^{2}-x^{2}-y^{2}
$$

(the Jordan circle product) and

$$
\{x y z\}:=V_{x, y} z:=U_{x, z} y:=\left(U_{x+z}-U_{x}-U_{z}\right) y
$$


(the Jordan triple product), the following identities hold in all scalar extensions:

$$
\begin{aligned}
V_{x, x} y & =x^{2} \circ y, \\
U_{x}(x \circ y) & =x \circ U_{x} y, \\
U_{x} x^{2} & =\left(x^{2}\right)^{2}, \\
U_{x} U_{y} x^{2} & =\left(U_{x} y\right)^{2}, \\
U_{x^{2}} & =U_{x}{ }^{2}, \\
U_{U_{x} y} & =U_{x} U_{y} U_{x} .
\end{aligned}
$$

Given a non-unital Jordan algebra $J$, a (Jordan) subalgebra of $J$ is a $k$-submodule stable under the operations $x^{2}$ and $U_{x} y$. An ideal of $J$ is a $k$-submodule $I \subseteq J$ such that $I^{2}+I \circ J+U_{I} J+U_{J} I+\{J J I\} \subseteq J$. An ideal $I \subseteq J$ is always a subalgebra, and the quotient $J / I$ is a non-unital Jordan algebra in a natural way. If $I_{1}, I_{2}$ are ideals in $J$, so is $U_{I_{1}} I_{2}$; if $U_{I_{1}} I_{2}=\{0\}$, then the ideals $I_{1}, I_{2}$ are said to be orthogonal.

Every unital Jordan algebra can be viewed as a non-unital Jordan algebra with squaring defined by (2.11). Obviously, unital subalgebras then become subalgebras also in the non-unital sense.

9.2. Unitizations. Given a non-unital Jordan algebra $J$ over $k$, let $k 1$ be a free $k$-module of rank 1 . Then the direct sum $\hat{J}:=J \oplus k 1$ becomes a unital Jordan algebra, the (free) unital hull of $J$, under the $U$-operator

$$
U_{x+\lambda 1}(y+\mu 1)=\left(U_{x} y+\mu x^{2}+2 \lambda \mu x+\lambda(x \circ y)+\lambda^{2} y\right)+\lambda^{2} \mu 1,
$$

making $J \subseteq \hat{J}$ an ideal 12 . By factoring out an ideal $I$ of $\hat{J}$ that is maximal with respect to the property of having trivial intersection with $J$ (which exists by Zorn's Lemma), we obtain the unital Jordan algebra $J^{\prime}:=\hat{J} / I$ (a tight unital hull of $J$ ) such that $J$ embeds as an ideal in $J^{\prime}$ in a tight way, meaning that any non-zero ideal of $J^{\prime}$ intersects $J$ non-trivially.

9.3. Extending results to the non-unital case. Since non-unital Jordan algebras can always be viewed as subalgebras of unital ones (9.2), many useful properties of the former retain their validity in the broader setting. In particular, Section 2.6 holds for arbitrary non-unital Jordan algebras, as do Propositions 5.1 and 5.2.

9.4. Non-degeneracy and primeness. Let $J$ be a non-unital Jordan algebra over $k$. An element $z \in J$ is an absolute zero divisor if $U_{z}=0$. We say $J$ is nondegenerate if it does not contain absolute zero divisors other than zero. There is a unique smallest ideal in $J$, called its McCrimmon radical and denoted by $\operatorname{Mc}(J)$, making the quotient $J / \operatorname{Mc}(J)$ non-degenerate. $J$ is said to be prime if it does not contain non-zero orthogonal ideals. Non-unital Jordan algebras that are both prime and non-degenerate are called strongly prime.

After these preparations, we will now be able to establish the main result of the paper.

Theorem 9.5. Let $J$ be a non-degenerate non-unital Jordan algebra over $k$. If $x, y \in J$ satisfy $x \circ y=0$, then $U_{x}$ and $U_{y}$ commute.

Proof. We carry out a number of reductions that will eventually allow us to make use of our preceding answers to question (111). First of all, by [20, Corollary 4], $J$ is a subalgebra of a direct product of strongly prime non-unital Jordan algebras. 
Hence we may assume that $J$ itself is strongly prime. Now let $J^{\prime}$ be a tight unital hull of $J$. By tightness, $J^{\prime}$ is also prime (non-zero orthogonal ideals of $J^{\prime}$ would give rise to non-zero orthogonal ideals of $J$ ) and non-degenerate (we have $\operatorname{Mc}(J)=0$, but also $\operatorname{Mc}(J)=\operatorname{Mc}\left(J^{\prime}\right) \cap J$ by [20, Corollary to Theorem 5]; hence $\operatorname{Mc}\left(J^{\prime}\right)=0$ by tightness). Thus we may assume that $J$ is unital. Now the Zel'manov-McCrimmon structure theory [15, 15.1,15.4] implies that $J$ is either special or an Albert form. Hence Section 4.1 and Theorem 8.1 yield the desired conclusion.

\section{ACKNOWLEDGEMENTS}

The authors are indebted to O. Loos and K. McCrimmon for illuminating comments. The authors give special thanks to Y. Segev for having drawn their attention to the problem of commuting $U$-operators in Jordan algebras, and for putting it in perspective with the theory of Moufang sets. The permission of Y. Segev and $\mathrm{K}$. Tent to quote one of their unpublished results in Section 3 is gratefully acknowledged.

Last but not least, the authors are greatly indebted to the referee, who studied the paper very carefully and, by making several useful suggestions, contributed significantly to an improved presentation of the subject.

\section{REFERENCES}

[1] Tom De Medts and Yoav Segev, A course on Moufang sets, Innov. Incidence Geom. 9 (2009), 79-122. MR 2658895 (2011h:20058)

[2] Tom De Medts and Richard M. Weiss, Moufang sets and Jordan division algebras, Math. Ann. 335 (2006), no. 2, 415-433, DOI 10.1007/s00208-006-0761-8. MR2221120 (2007e:17027)

[3] John R. Faulkner, Octonion planes defined by quadratic Jordan algebras, Memoirs of the American Mathematical Society, No. 104, American Mathematical Society, Providence, R.I., 1970. MR0271180 (42 \#6063)

[4] John R. Faulkner, Finding octonion algebras in associative algebras, Proc. Amer. Math. Soc. 104 (1988), no. 4, 1027-1030, DOI 10.2307/2047585. MR931729 (89g:17003)

[5] Nathan Jacobson, Structure and representations of Jordan algebras, American Mathematical Society Colloquium Publications, Vol. XXXIX, American Mathematical Society, Providence, R.I., 1968. MR0251099 (40 \#4330)

[6] Nathan Jacobson, Lectures on quadratic Jordan algebras, Tata Institute of Fundamental Research, Bombay, 1969, Tata Institute of Fundamental Research Lectures on Mathematics, No. 45. MR0325715 (48:4062)

[7] Nathan Jacobson, Structure theory of Jordan algebras, University of Arkansas Lecture Notes in Mathematics, vol. 5, University of Arkansas, Fayetteville, Ark., 1981. MR634508 (83b:17015)

[8] Serge Lang, Algebra, 3rd ed., Graduate Texts in Mathematics, vol. 211, Springer-Verlag, New York, 2002. MR.1878556(2003e:00003)

[9] Robert E. Lewand and Kevin McCrimmon, Macdonald's theorem for quadratic Jordan algebras, Pacific J. Math. 35 (1970), 681-706. MR0299648 (45 \#8696)

[10] Kevin McCrimmon, The Freudenthal-Springer-Tits constructions of exceptional Jordan algebras, Trans. Amer. Math. Soc. 139 (1969), 495-510. MR0238916 (39 \#276)

[11] Kevin McCrimmon, The Freudenthal-Springer-Tits constructions revisited, Trans. Amer. Math. Soc. 148 (1970), 293-314. MR0271181 (42 \#6064)

[12] Kevin McCrimmon, Quadratic Jordan algebras and cubing operations, Trans. Amer. Math. Soc. 153 (1971), 265-278. MR.0268239 (42 \#3138)

[13] Kevin McCrimmon, Nonassociative algebras with scalar involution, Pacific J. Math. 116 (1985), no. 1, 85-109. MR769825 (86d:17003)

[14] Kevin McCrimmon, A taste of Jordan algebras, Universitext, Springer-Verlag, New York, 2004. MR2014924 (2004i:17001) 
[15] Kevin McCrimmon and Ephim Zel'manov, The structure of strongly prime quadratic Jordan algebras, Adv. in Math. 69 (1988), no. 2, 133-222, DOI 10.1016/0001-8708(88)90001-1. MR.946263(89k:17052)

[16] Holger P. Petersson, An embedding theorem for reduced Albert algebras over arbitrary fields, to appear in Communications in Algebra.

[17] Holger P. Petersson, On linear and quadratic Jordan division algebras, Math. Z. 177 (1981), no. 4, 541-548, DOI 10.1007/BF01219086. MR624231 (82h:17013)

[18] Holger P. Petersson and Michel L. Racine, Jordan algebras of degree 3 and the Tits process, J. Algebra 98 (1986), no. 1, 211-243, DOI 10.1016/0021-8693(86)90024-4. MR.825144 (87h:17038a)

[19] Tonny A. Springer and Ferdinand D. Veldkamp, Octonions, Jordan algebras and exceptional groups, Springer Monographs in Mathematics, Springer-Verlag, Berlin, 2000. MR1763974 (2001f:17006)

[20] Armin Thedy, z-closed ideals of quadratic Jordan algebras, Comm. Algebra 13 (1985), no. 12, 2537-2565, DOI 10.1080/00927878508823290. MR811523 (87a:17026)

[21] Jacques Tits and Richard M. Weiss, Moufang polygons, Springer Monographs in Mathematics, Springer-Verlag, Berlin, 2002. MR1938841 (2003m:51008)

Departamento de Matemáticas, Universidad de Oviedo, E-33007 Oviedo, Spain

E-mail address: anque@orion.ciencias.uniovi.es

Departamento de Matemáticas, Universidad de Oviedo, E-33007 Oviedo, Spain

E-mail address: cortes@orion.ciencias.uniovi.es

Fakultät Für Mathematik und Informatik, FernUniversität in Hagen, D-58084 Hagen, Germany

E-mail address: holger.petersson@fernuni-hagen.de 